\title{
Review of Fusion Research Program: Historical Summary and Program Projections
}

by

E. S. Murphy

September 1976

Prepared for the Energy Research and Development Administration under Contract E(45-1)-1830 


\section{NOTICE}

This report was prepared as an account of work sponsored by the United States Government. Neither the United States nor the Energy Research and Development Administration, nor any of their employees, nor any of their contractors, subcontractors, or their employees, makes any warranty, express or implied, or assumes any legal liability or responsibility for the accuracy, completeness or usefulness of any information, apparatus, product or process disclosed, or represents that its use would not infringe privately owned rights.

PACIFIC NORTHWEST LABORATORY

operated by

BATTELLE

for the

ENERCY RESEARCH AND DEVELOPMENT ADMINISTRATION

Under Contract EY-76-C-06-1830

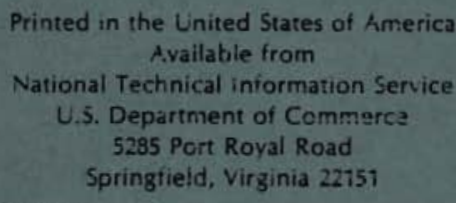

Price: Printed Copys $\because$ Microfiche $\$ 3.00$

$\begin{array}{cc}\text {-Pages } & \begin{array}{c}\text { NTIS } \\ \text { Selling Price }\end{array} \\ 001-025 & \$ 4.50 \\ 026-050 & \$ 5.00 \\ 051-075 & \$ 5.50 \\ 076-100 & \$ 6.00 \\ 101-125 & \$ 6.50 \\ 126-150 & 57.00 \\ 151-175 & 57.75 \\ 176-200 & \$ 8.50 \\ 201-225 & \$ 8.75 \\ 226-250 & 59.00 \\ 251-275 & \$ 10.00 \\ 276-300 & 510.25\end{array}$




\section{1}

REVIEW OF FUSION RESEARCH PROGRAM: HISTORICAL SLMMARY AND PROGRAM PROJECTIONS

by

E. S. Murphy

September 1976

BATTELLE

Pacific Northwest Laboratories

Richland, Washington 99352 


\section{PREFACE}

Fusion reactor technology has developed far enough to expect laboratory demonstration of practical levels of fusion employing the D-T reaction to occur in the early 1980s. Following that demonstration, and depending upon the national priorities for energy from $D-T$ fusion, construction and operation of experimental reactors and demonstration power reactors could occur before the end of this century. Operation of the first commercial power plants could then follow, starting about 2010.

Development and adoption of a new power system eventualiy will require a description of the environmental effects in an environmental statement providing a comparison to the effects of competitive systems. In anticipation of that statement, an environmental analysis (BNWL-2010) has been prepared for the ERDA Division of Magnetic Fusion Energy. That analysis estimates the environmental effects of constructing and operating D-T fusion reactors as an economically competitive source of electricity in the 21 st century.

The analysis has four primary purposes:

1. To describe the general nature of the environmental effects,

2. To determine current ability to estimate the effects,

3. To determine methods for reducing the effects, and

4. To determine research necessary for increasing capability to define and reduce the effects.

Timely identification of needed research and methods for reducing effects will permit the performance of that research and the revision of conceptual fusion power plant designs before preparation of the program environmental statement. This would improve the quality of the environmental statements and could reduce the estimated adverse environmental effects due to fusion power plants.

The environmental analysis (BNWL-2010) concludes that the following assumed characteristics are the best set for the first operating fusion power plants:

- The D-T fusion reaction

- Large quantities of activation products

- Kilogram quantities of tritium in the plant systems

- Massive reactor structures

- Large lithium inventories

- Large inventories of liquid metals and salts
Standard electricity generation Standard radioactive waste systems Large magnetic fields A self-contained fuel cycle Rural siting

Using these characteristics a reference reactor was analyzed to determine the environmental effects by using available concepts of plant subsystems designs that control interactions with the environment or by assumption that best current technology would be used in subsystems design. Because this analysis does not take into account advances in both fusion and waste control technology during the next thirty years, the estimated effects probably are significantly higher than the actual effects will be for the first fusion power plants. The estimated environmental effects should be interpreted only as being the probable upper limit for the actual effects. 
Preparation of the fusion power plant environmental analys is required development and use of specially developed data and analysis methods not used in the preparation of current environmental statements for fossil and fission power plants. These data and analysis requirements are documented in a series of reference topical reports to make this information publicly available and to assure understanding of the basis for the conclusions made in the environmental analysis.

These reference topical reports sumarize the state-of-the-art as applicable to preparation of environmental statements for fusion power plants. They present the data and analytical techniques used in the environmental analysis to estimate the interactions with the environment and the resultant environmental effects. This information then was analyzed for adequacy and the need was determined for additional research to assure satisfactory ability to prepare environmental statements for the fusion development program and experimental facilities in the early 1980s. Estimated environmental effects are presented in these reference documents only as necessary to illustrate use of the data and analytical techniques.

This report is one of those reference documents for the environmental analysis. The other documents in this series contain more details of the power plant concepts and the probable environmental effects of fusion power plants with the assumed characteristics listed above. These documents are available through the National Technical Information Service:

An Environmental Analysis of Fusion Power to Determine Related R\&D Needs, BNWL-2010

Review of Fusion Research Program: Historical Sumary and Program Projections, BNWL-201 1

Fuel Procurement for First Generation Fusion Power Plants, BNWL-2012

Current Fusion Power Plant Design Concepts, BNHL-2013

Reference Cormerical Fusion Power Plants, BNWL-2014

Siting Commercial Fusion Power P1ants, BNWL-2015

Materials Availability for Fusion Power Plant Construction, 8NWL-2016

Projected Thermodynamic Efficiencies of Fusion Power Plants, BNWL-2017

Tritium Source Terms for Fusion Power Plants, BNWL-2018

Management of Nontritium Radioactive Wastes from Fusion Power Plants, BNWL-2019

Methodology for Estimating Radiation Doses Due to Tritium and Radiocarbon Releases, BNWL-2020

Magnetic Field Considerations in Fusion Power Plant Environs, BNWL-2021

Biological Effects of Tritium Releases from Fusion Power Plants, BNWL-2022

Biological Effects of Activation Products and Otner Chemicals Released from Fusion Power Plants, BNWL-2023

Safety Review of Conceptual Fusion Power Plants, BNWL-2024

An Investigation of the Transportation Requirements of Fusion Power Plants, BNWL-2025

Considerations of the Social Impact of Fusion Power, BNWL-2026

Environmental Impacts of Nonfusion Power Systems, BNWL-2027

Environmental Cost/Benefit Analysis for Fusion Power Plants, BNWL-2028

Biomagnetic Effects: A Consideration in Fusicn Reactor Development, BNWL-1973

An Analysis of Tritium Releases to the Atmosphere by a CTR, BNWL-1938 
CONTENTS

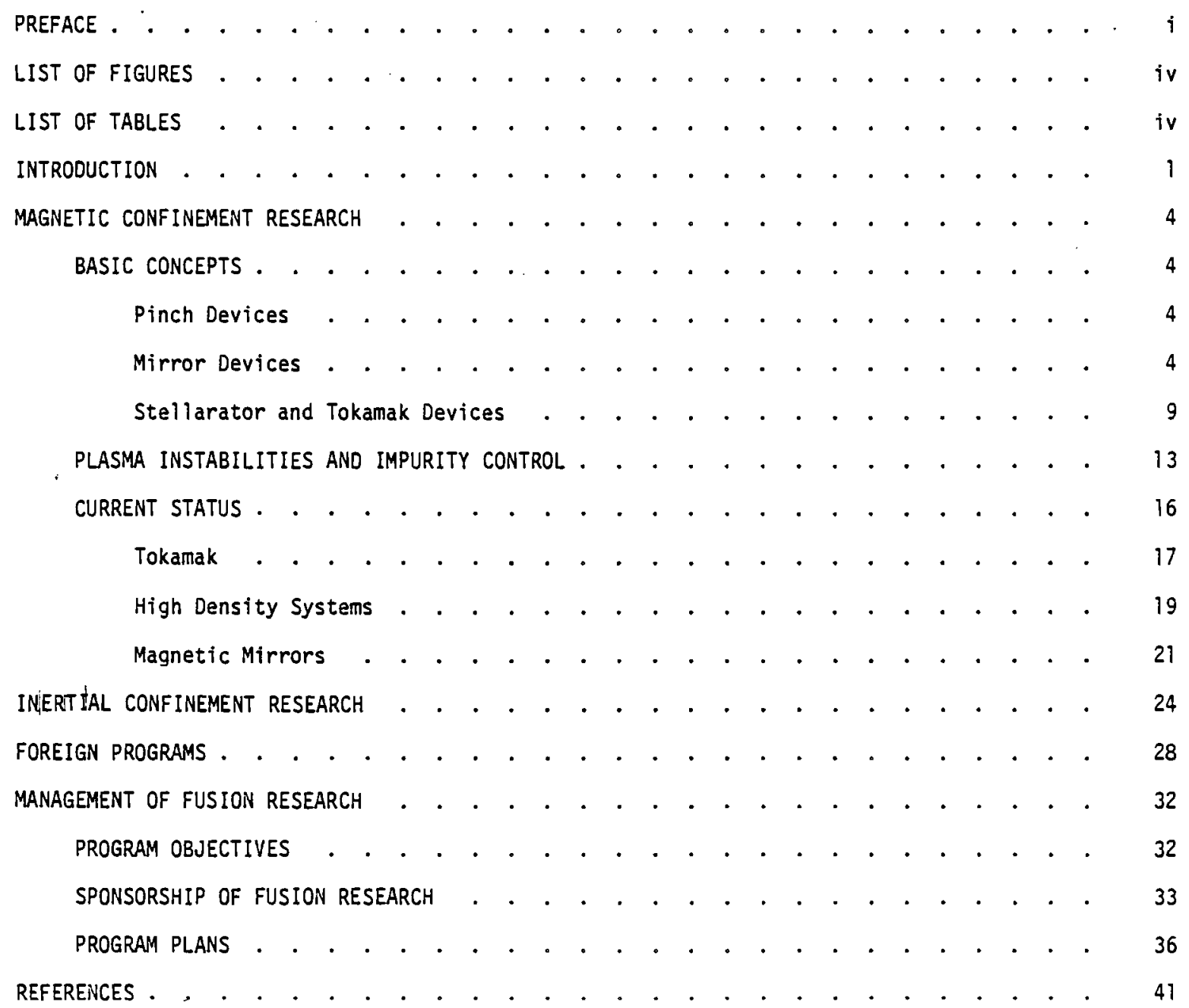




\section{LIST OF FIGURES}

1 Self Induced Pinch of a Toroidally. Confined Plasma . . . . . . . . . . . . 4

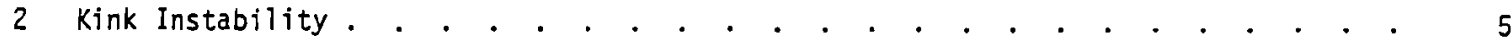

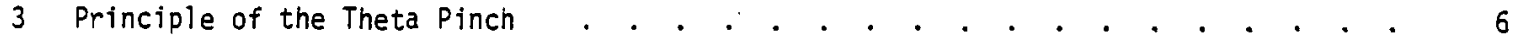

4 Helical Paths of Ions and Electrons in a Magnetic Field . . . . . . . . $\quad 7$

5 Simple Magnetic Mirror . . . . . . . . . . . . . . . . . . . . . 8

6 Interlocking Field Coils Used to Produce a Magnetic Well in the $2 X$-II Device . . $\quad 8$

7 Magnetic Well in Baseball-II Produced by Field Coils Shaped Like the Seam on

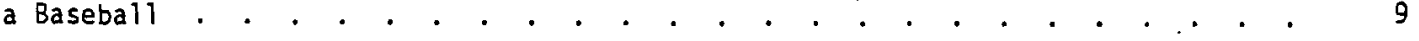

8 Confinement of a Plasma in a Simple Torus . . . . . . . . . . . . . . . . . 10

9 Helical Magnetic Field Lines Possessing the Property of Shear . . . . . . . . 11

10 Schematic of Tokamak Device . . . . . . . . . . . . . . . . . . . . . 12

11 Plasma Density Decay Curves for the 2 X Machine . . . . . . . . . . . . . 15

12 Progress in Attainment of Fusion Reactor Conditions . . . . . . . . . . . 17

13 Toroidal Sector of Scyllac Machine . . . . . . . . . . . . . . . . . 20

$142 X-I I$ Magnetic Confinement Device . . . . . . . . . . . . . . . . . . 22

15 Proposed inf Facility . . . . . . . . . . . . . . . . . . . . . . 23

\section{LIST OF TABLES}

1 Fusion Reactions of Interest . . . . . . . . . . . . . . . . . . . 1

2 Major Tokamak Experiments Which Have Been or Are Now in Operation . . . . . . 12

in the United States $\cdot \cdot \cdot \cdot \cdot \cdot \cdot \cdot \cdot \cdot \cdot \cdot \cdot \cdot \cdot \cdot \cdot \cdot \cdot \cdot \cdot \cdot \cdot \cdot 12$

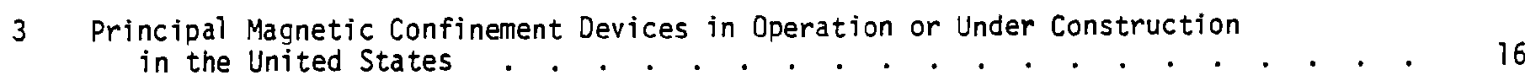

4 Existing Laser Systems at Government-Sponsored Reserach Laboratories . . . . . 26

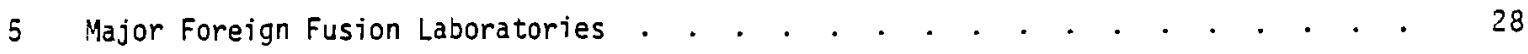

$6 \quad$ Large Tokamak Experiments Currently Planned or Under Construction Worldwide . . 31

7 Federal Budgets for Magnetic Confinement Research for the Years 1951 - 1976 • • • 35

8 Federal Budgets for Inertial Confinement Research for the Years $1963-1976$. . . 36

9 Proposed Characteristics of Devices in the Long-Range Hagnetic Confinement

Program Leading to Commercialization of Fusion Power . . . . . . . . . . 38 
REVIEW OF FUSION RESEARCH PROGRAM:

HISTORICAL SUMMARY AND PROGRAM PROJECTIONS

\section{INTRODUCTION}

This report provides a brief review of the history and current status of fusion research in the United States. It also describes the federally funded program aimed at the development of fusion reactors for electric power generation.

Development of new sources of unlimited energy which are environmentally acceptable is one of the most critical technical challenges facing our nation in the last quarter of the twentieth century. Among such energy sources, the production of electricity from fusion ranks high as a possible provider of our nation's longterm energy requirements. The goal of the federally funded fusion power research and development program is to demonstrate, before the year 2000, fusion power electric generating stations for comercial application.

Recent progress in fusion research has brought the fusion power program to a point where a majority of its research and development activities can be reasonably well defined. Therefore, meaningful program planning has become possible and target dates for construction and operation of major fusion facilities have been established. Short term objectives of the fusion power research and development program center around establishing the technical. feasibility of the more promising concepts which could best lead to commercial power systems. Longterm program objectives include milestones for the operation of experimental fusion power devices culminating, before the year 2000, in a Demonstration Power Reactor which would provide the basis for commercialization of fusion power.

Fusion reactions occur when two light nuclei react to form product nuclei having a smaller total mass than that of the original nuclei. In such reactions there is a release of energy. Typical fusion reactions of interest to controlied thermonuclear research include those shown in Table 1.

Table 1. Fusion Reactions of Interest

\begin{tabular}{cc} 
Reaction & Energy Released \\
\hline$D+T+{ }^{4} \mathrm{He}+n$ & $17.6 \mathrm{MeV}$ \\
$D+D+{ }^{3} \mathrm{He}+\mathrm{T}+p$ & $3.3 \mathrm{MeV}$ \\
$0+{ }^{3} \mathrm{He}+{ }^{4} \mathrm{He}+p$ & $4.0 \mathrm{MeV}$ \\
${ }^{6} \mathrm{Li}+\mathrm{P} \rightarrow{ }^{3} \mathrm{He}+{ }^{4} \mathrm{He}$ & $18.3 \mathrm{MeV}$
\end{tabular}

The fuel atoms in fusion devices are totally ionized, i.e., broken down to their elementary constituents of positive ions (atomic nuclei) and electrons. Such a totally ionized gas is called a plasma. In order for the positive ions in the plasma to come together and react, they 
must have sufficient kinetic energy to overcome the Coulomb forces tending to produce separation. This requires that the plasma be heated to a temperature of the order of millions of degrees Kelvin. At very high temperatures some of the particles will fuse, but other particles will undergo scattering interactions and lose energy by radiative processes. The rates of both energy production due to fusion and energy loss due to radiative processes increase with temperature, but the rate of energy production increases faster with the result that at a certain critical ignition temperature the fusion reaction becomes self-sustaining. For the deuteriumtritium (D-T) reaction the ignition temperature is about 50 million degrees Kelvin. Since the D-T reaction has the lowest ignition temperature of any of the reactions listed in Table 1 , it is the one which has received the most attention in planning for the first fusion power plants.

In addition to being maintained at a very high temperature, the plasma in a practical fusion reactor must be of sufficient density and must be confined for a long enough time so that the energy produced from fusion reactions exceeds the energy input required to confine and heat the plasma. Plasma confinement can be stated in terms of the product of particle density ( $n$ ) and particle confinement time $(\tau)$. For net energy gain, the $n \tau$ product of a system must exceed a minimum value called the Lawson criterion. For a deuterium-tritium system the Lawson criterion is $n \tau \approx 10^{14} \mathrm{~cm}^{-3} \mathrm{sec}$. An important density confinement time tradeoff is possible. A plasma with density of $10^{14} \mathrm{~cm}^{-3}$ must be confined for 1 second, but a plasma with density of $10^{15} \mathrm{~cm}^{-3}$ need only be confined for 0.1 second.

The attainment of practical fusion power depends on achieving the necessary temperature, density and confinement time in a suitably confined plasma. Confinement of the plasma in a material vessel will not work because particle collisions with the walls of the vessel result in energy losses which imediately cool the plasma below the fusion temperature. Cooling is accelerated by a buildup in the plasma of impurity atoms dislodged by collision from the walls of the containment vessel.

Experiments begun independently and almost simultaneously about 1951 in the United $S$ tates, the United Kingdom, and the USSR have had as their goal the confinement of a plasma by a magnetic field. A number of different field configurations for plasma confinement have been investigated. Early optimism for success of the magnetic confinement program was replaced by restraint as plasma. instabilities prevented attainment of confinement conditions necessary for reactor operation. For over two decades the major effort in fusion research has been the attempt to understand and overcome plasma instabilities in magnetic confinement devices. The success of this effort and the major experimental devices which have resulted will be described in the sections which follow. Magnetic confinement research is managed by the Division of Magnetic Fusion Energy (formerly the Division of Controlled Thermonuclear Research) of the Energy Research and Development Administration (ERDA).

Since 1960, an alternative method of plasma confinement involving implosion and heating of a D-T pellet by high-energy laser or electron beams has been the subject of extensive theoretical investigation. This technique, called inertial confinement, would use pellet irradiation by very high power laser or electron beams to achieve plasma densities that will reach approximately 
$10^{26} \mathrm{~cm}^{-3}$. The plasma confinement time (about $10^{-12}$ seconds) would be determined by the inertia of matter. As described in succeeding sections, experimental verification of inertial confinement has not yet been achieved. Major experimental programs are under way both in the United States and abroad to develop the laser and electron beams needed for verification of the inertial confinement principle. All inertial confinement research was, until recently, managed by the Division of Military Application of the ERDA. Recent administrative changes have created a Division of Laser Fusion for management of commercially oriented inertial confinement programs. 


\section{MAGNETIC CONFINEMENT RESEARCH}

\section{BASIC CONCEPTS}

The history of magnetic confinement research in the United States, for the period 1951-1958, is described in Reference 1. Recent developments are described in several journal articles. (2-10)

Magnetic confinement research in the United States has focused on three concepts. In terms of plasma densities and geometrical configurations, these concepts can be described as high density systems ( $Z$-pinch and theta pinch devices); low density open systems (magnetic mirrors); and low density closed systems (stellarators and tokamaks).

\section{Pinch Devices}

Pinch devices utilize strong magnetic fields to compress a plasma and increase its temperature. They are usually pulsed devices and have been constructed in both linear and toroidal configurations. The first pinch devices, constructed at LoS Alamos Scientific Laboratory (LASL) in the early 1950s under the direction of 3 . L. Tuck, were Z-pinch machines. In Z-pinch machines the confining magnetic field is produced by internal currents within the plasma itself. Interaction of the plasma current with its own magnetic field resuits in an inwardly directed force which tends to constrict or "pinch" the plasma (Figure 1) producing an increase in both plasma density and piasma temperature.

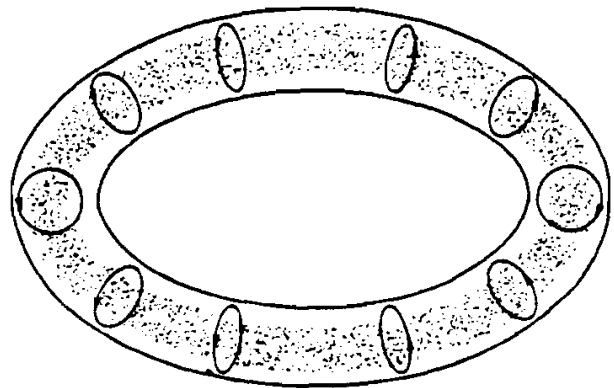

START OF THE DISCHARGE

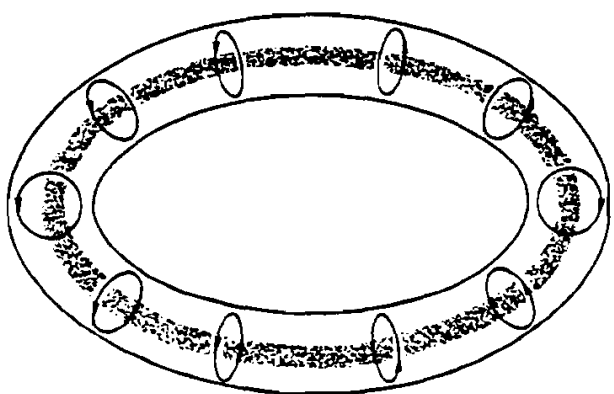

FULLY FORMED PINCH

FIGURE 1. Self Induced Pinch of a Toroidally Confined Plasma 
Densities and confinement times of early pinch devices were many orders of magnitude below the requirements of a fusion reactor. The inability of pinch devices to produce adequate confinement times was due to plasma instabilities, the most serious of which, for $z$-pinch machines, was the so-called "kink" instability. The kink instability, shown in Figure 2, arises when a small bend develops in a confined column of plasma. The density of self-induced magnetic field lines is greater on the concave side of the kink than it is on the convex side of the kink. This results in a net outward force which causes the kink to grow in size. The plasma quickly strikes the wall of the vacuum chamber and dissipates its energy, thus cooling the discharge.

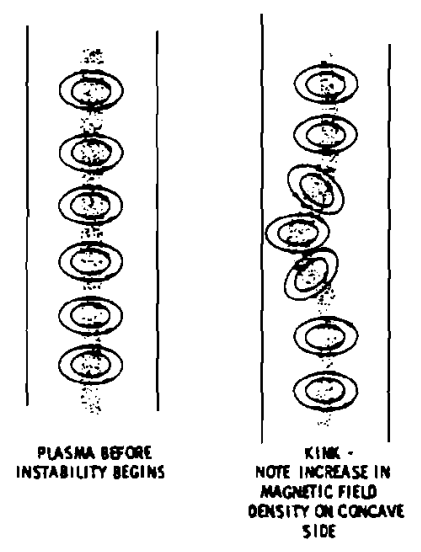

\section{FIGURE 2. Kink Instability}

In 1957, theta pinch research was initiated at LASL with construction of the first machine, termed a Scylla. In theta pinch devices a pulse of current in the azimuthal (theta) direction produces a fast rising axial magnetic field. The fast rising magnetic field induces currents in the plasma by transformer action, which flow in the "theta" direction encircling the coil axis. Interaction of these plasma currents with the rising magnetic field heats and compresses the plasma. Theta pinch machines are pulsed devices which attempt to produce a very rapid reduction in plasma volume where a significant number of fusion reactions will occur before the onset of instability. Figure 3 shows the plasma in a theta pinch machine being compressed (dynamic phase), and the compressed plasma (quiescent phase).

At LASL both linear (Scylla) and toroidal (Scyllac) theta-pinch devices have been constructed. Early research was performed on linear devices and showed that: 1) ion heating to thermonuclear temperatures ( 3 to $5 \mathrm{keV}$ ) could be routinely achieved in theta pinch geametry, and 2) loss of plasma from the ends of the linear theta-pinch device was the limiting factor on confinement.

Because the extrapolated length of a linear theta-pinch device with a confinement time suitable for fusion reactor operation would make the device impractically long, scientists at LASL began in the mid-1960s to investigate toroidal theta-pinch machines. Success with toroidal sectors led to initial operation of a full torus Scyllac device in mid-1974. Experiments are currently underway to demonstrate plasma stability in the full torus Scyllac. 


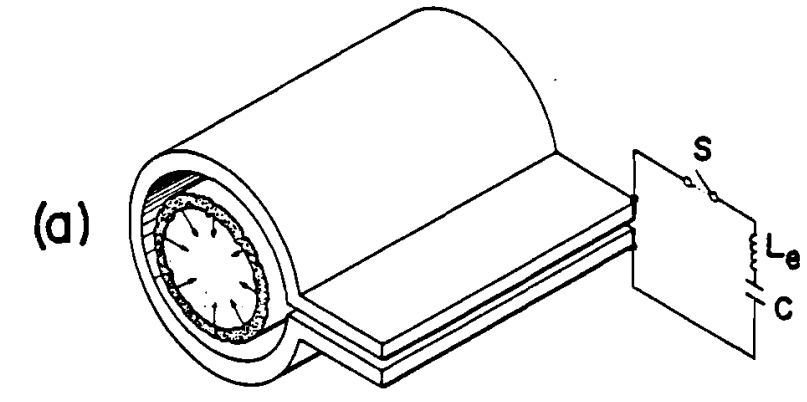

DYNAMIC PHASE

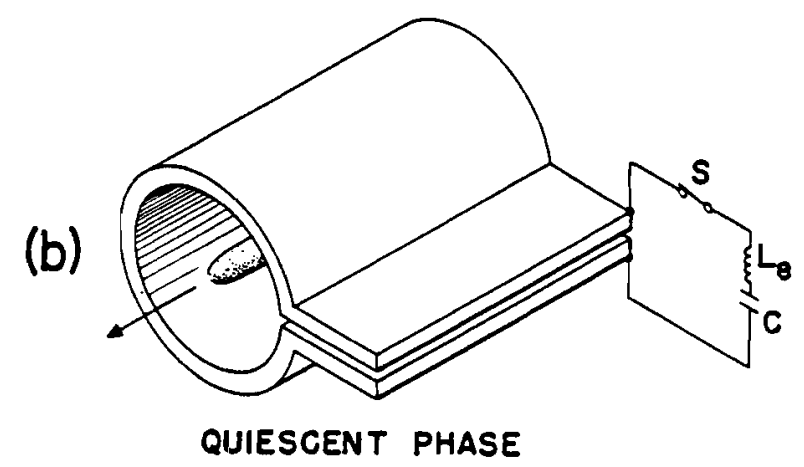

FIGURE 3. Principle of the Theta Pinch

To produce the intense pulsed magnetic fields needed for plasma compression in theta-pinch machines, large amounts of energy are required. The Scyllac Toroidal Sector at LASL utilized a $14 \mathrm{~cm}$ diameter one-turn coil powered by a $60 \mathrm{kV}, 3.6 \mathrm{MJ}$ capacitor bank. The bank was switched by spark gaps, resulting in a magnetic field rise time of $5 \mu \mathrm{sec}$.

Present theta-pinch devices derive the energy for ion heating from a single large capacitor bank. However, future machines will utilize a concept, called staging, in which separate systems will provide the energy for a fast rising (microseconds) shock-heating field and a slower rising (milliseconds) field which adiabatically compresses the plasma to reactor temperatures and densities. The basic concepts of staging are currently being tested in a Staged Theta Pinch experiment which began operation at LASL in 1975.

\section{Mirror Devices}

In a magnetic field, plasma particles (positive ions and electrons) travel in helical paths as shown in Figure 4. The simplest field configuration for magnetic confinement of a plasma is a linear homogeneous field. In such a field, plasma particles will not move across field lines toward vessel walls except as a result of collisions with other particles which change their direction of motion. At plasma densities common for fusion devices $\left(210^{-5}\right.$ atmospheres for low density systems) the time for collisional loss of the plasma, called the classical confinement time, is large compared with the confinement time needed to achieve net energy production from a fusion reactor. 


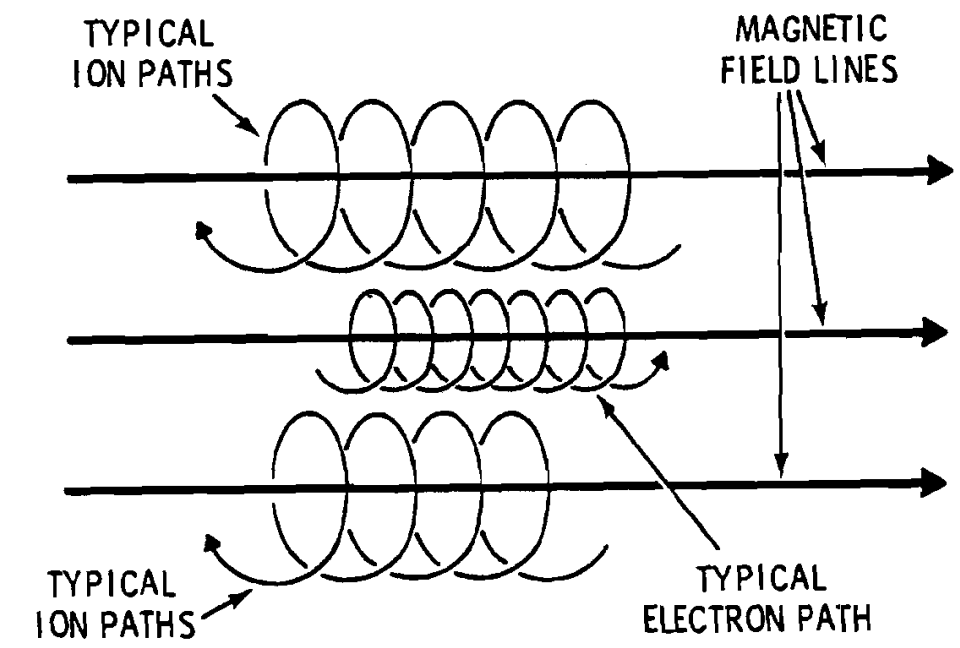

FIGURE 4. Helical Paths of Ions and Electrons in a Magnetic Field

Because plasma particles will escape from the ends of a device of finite length, a linear homogeneous field cannot be used for steady state operation. In magnetic mirror devices (Figure 5), additional current carrying coils are utilized at the ends of the device to increase the field strength and reflect.escaping particles back into the plasma region from which they came. This effect may be likened to reflection by a mirror--hence, a localized high-field region of a magnetic field may be termed a magnetic mirror. Charged particle confinement by trapping between two magnetic mirrors is called mirror confinement.

A charged particle whose direction of motion is close enough to the direction of the magnetic field line around which it spirals will penetrate a magnetic mirror. Consequently, for each magnetic mirror there exists a "loss cone" through which particles can escape. Because plasma particles are continually scattered into the loss cone by collisions, mirror confinement is inherentiy leaky.

A program in magnetic mirror confinement of a plasma was initiated in 1952 at the University of California Radiation Laboratory at Livermore under the direction of H. F. York and R. F. Post. As had occurred with early toroidal pinch devices, the early magnetic mirror devices failed to confine the plasma for periods longer than microseconds. In simple mirror machines (Figure 5 ) the field lines are not linear, but instead bulge outward with distance from the axis of the machine. Al though increasing toward each mirror, the magnetic field decreases in the radial direction. The result is rapid escape of the plasma radially outward to the walls of the vacuum chamber.

In 1958, H. Grad and M. Leveine of New York University proposed on theoretical grounds that extra current carrying structures be added to mirror machines to produce a magnetic well* in which the plasma would be confined. The concept was successfully used in experiments with mirror machines by $M$. S. Ioffe and his coworkers in the USSR in 1961. (11)

\#A magnetic well is a region of minimum field strength from which the magnetic field increases in all directions. 


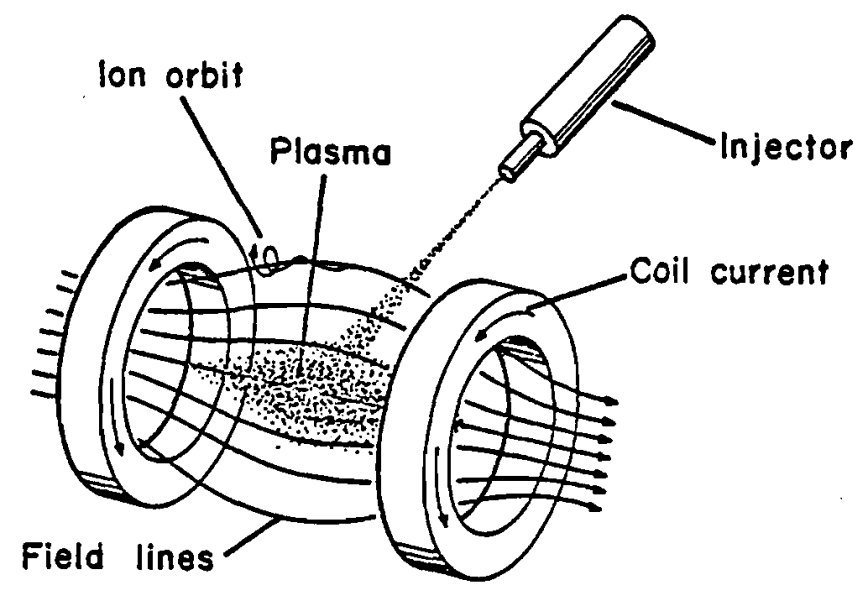

FIGURE 5. Simple Magnetic Mirror

Contemporary machines at Lawrence Livermore Laboratory (LLL) which employ the magnetic well concept include the $2 X-I I B$ device which uses two curved, flattened interlocking field coils called Yin Yang coils (Figure 6 ) and the Baseball IIT device which uses a superconducting field coil shaped like the seam of a baseball (Figure 7). The principal experimental device for magnetic mirror research in the United States is the 2X-IIB machine at Lawrence Livermore Laboratory. This device has regularly achieved ion temperatures of 1 to $10 \mathrm{keV}$ with plasma densities in the $10^{13}$ to $10^{14} \mathrm{~cm}^{-3}$ range. Confinement time has been shown to be "classical", i.e., to agree with theoretical predictions for the plasma volume and magnetic field strength used in the experiments.

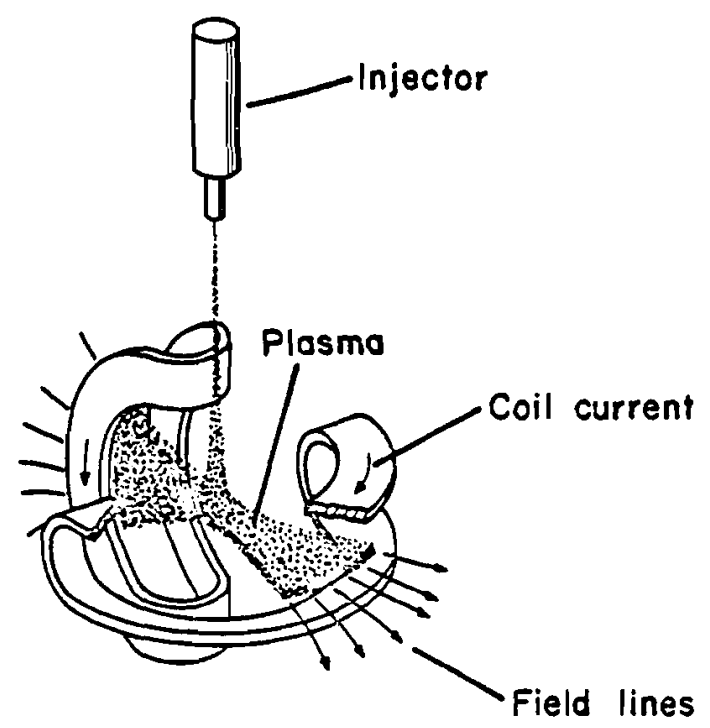

FIGURE 6. Interlocking Field Coils Used to Produce a Magnetic Well in the 2X-IIB Device 


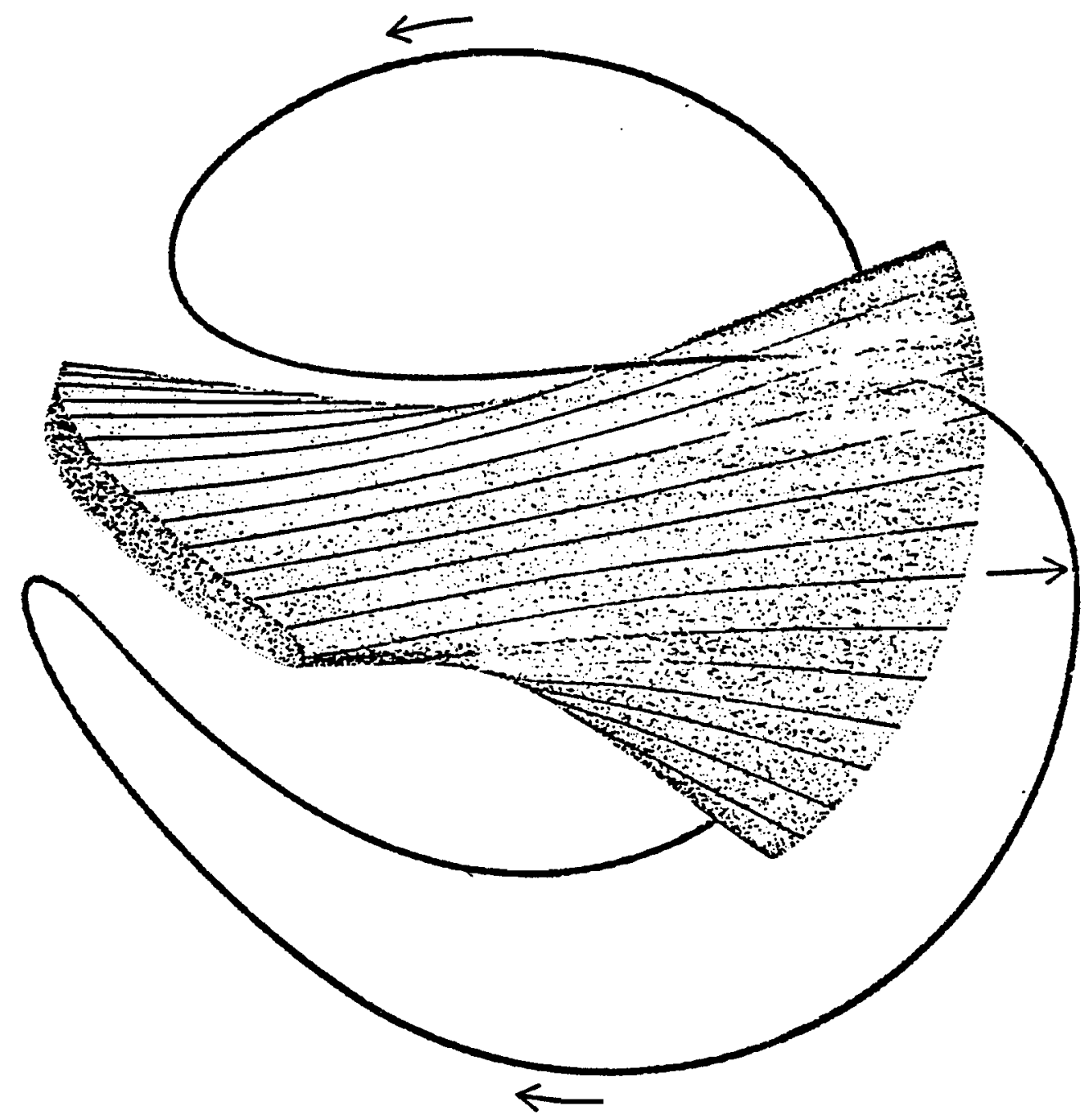

FIGURE 7. Magnetic Well in Baseball-IIT Produced by Field Coils Shaped Like the Seam on a Baseball

\section{Stellarator and Tokamak Devices}

An al ternative approach to the mirror concept of steady state plasma confinement is confinement in an endless tube produced by winding magnetic field coils in the shape of a torus. The concept is shown schematically in Figure 8. In 1951, at Princeton University, experiments a imed at plasma confinement by a doughnut shaped field were initiated under the direction of L. Spitzer, Jr. The machines which were built at Princeton Plasma Physics Laboratory (PPPL) to study steady state toroidal confinement were called stellarators. 
The magnetic field strength inside a simple torus is not uniform, being strongest at the inner edge of the torus and decreasing in strength with increasing distance from the toroidal axis. In this non-uniform field, charged particles do not follow simple spiral paths, but tend instead to drift upward or downward. The charge separation thus produced gives rise to an electric field which neutralizes the effect of the magnetic field and permits plasma particles to escape to the walls of the container. Charge separation in a stellarator-type machine can be prevented by altering the magnetic field so that the field lines do not close on themselves (i.e., by producing a magnetic field with a helical shape). Early stellarator machines achieved helical shaped field lines by twisting the torus to form a simple figure eight.

The plasma stability expected for the figure eight stellarator did not prove to be experimentally attainable. In large measure, this was shown to be due to magnetohydrodynamic (MHD) instabilities involving gross fluid-like motion of the plasma. (4) To overcome MHD instabilities in toroidal confinement devices it is necessary to give the magnetic lines of force a twist which is larger near the walls of the torus than it is close to the axis. Figure 9 shows a set of helical field lines possessing this characteristic. The sheared magnetic field shown in figure 9 prevents the growth of gross fluid-like instabilities since a perturbation which follows the lines of force on one magnetic surface no longer follows the lines on the next surface because the lines have shifted.

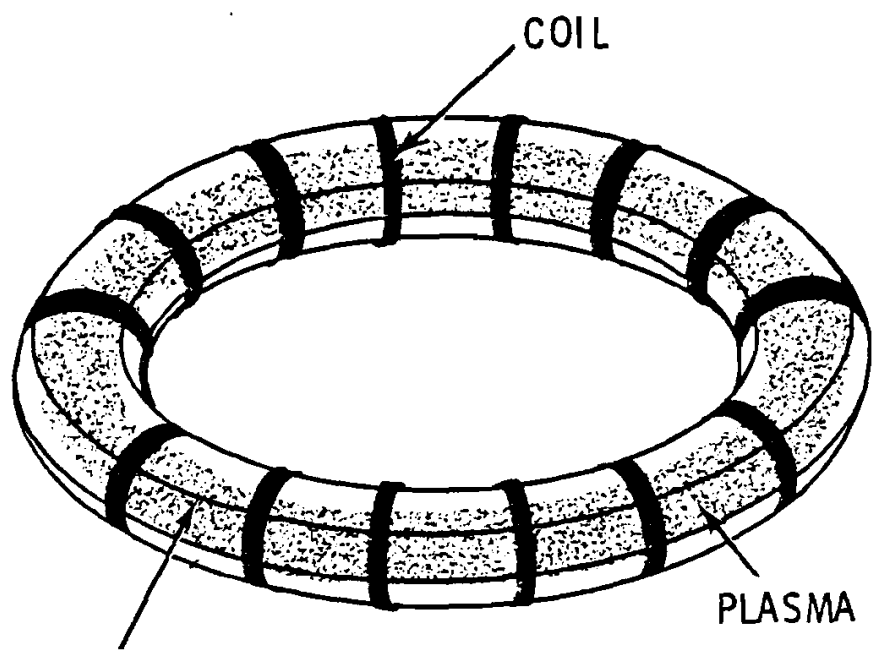

FIELD LINES

FIGURE 8. Confinement of a Plasma in a Simple Torus 


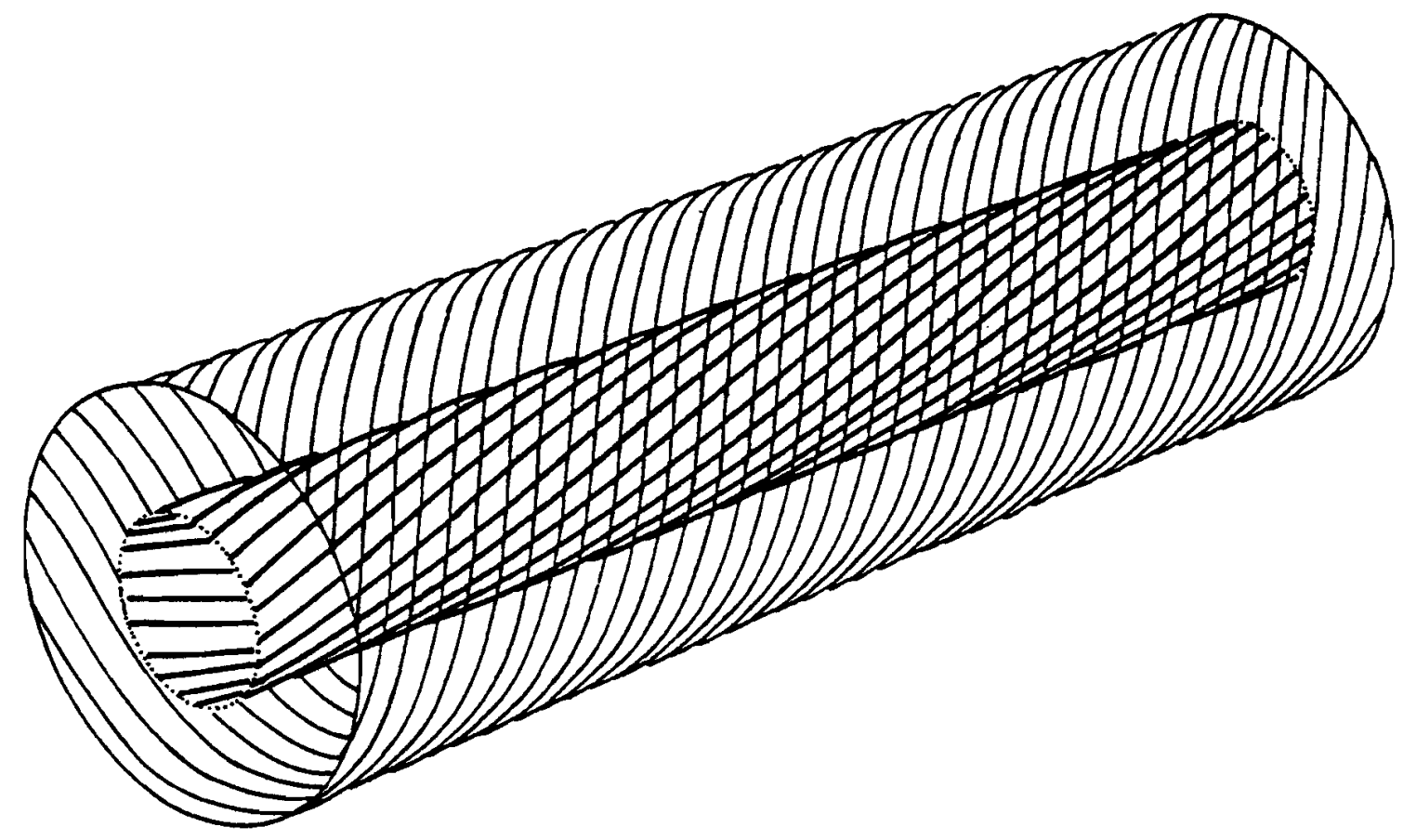

FIGURE 9. Helical Magnetic Field Lines Possessing the Property of Shear

In contemporary stellarator machines the geometrical configuration is again that of the simple torus. The confining field, having the properties shown in figure 9 , is produced by adding a set of specially shaped helical coils which are located inside the toroidal field coils.

An alternative method of generating the shear field necessary for plasma stabilization in a toroidal machine was developed by scientists at the I. V. Kurchatov Institute near Moscow during the late 1960s. The Russian device, called a tokamak, is shown schematically in Figure 10. The helical magnetic field which confines the plasma in a tokamak is actually the resultant of two fields which are oriented at right angles to each other. The toroidal field is produced by electric current flowing in coils distributed around the toroidal chamber. The poloidal field is generated by a large circulating current induced in the plasma itself by transformer action. As shown in Figure 10, the primary windings of the transformer are wound around a transformer core and the plasma serves as the secondary winding.

The inherent simplicity of the tokamak design and the improved confinement times demonstrated by tokamak devices have led to the abandonment of stellarator research in the United States in favor of the tokamak concept. Following the 1968 announcement of the Russian success with model T-3 tokamak, the model $C$ stellarator at PPPL was quickiy converted to the model ST tokamak. In recent years a variety of plasma confinement and heating experiments based on the tokamak concept have been undertaken in the United States. A list of the major tokamak devices which have been or are now in operation in the US is given in Table 2. 


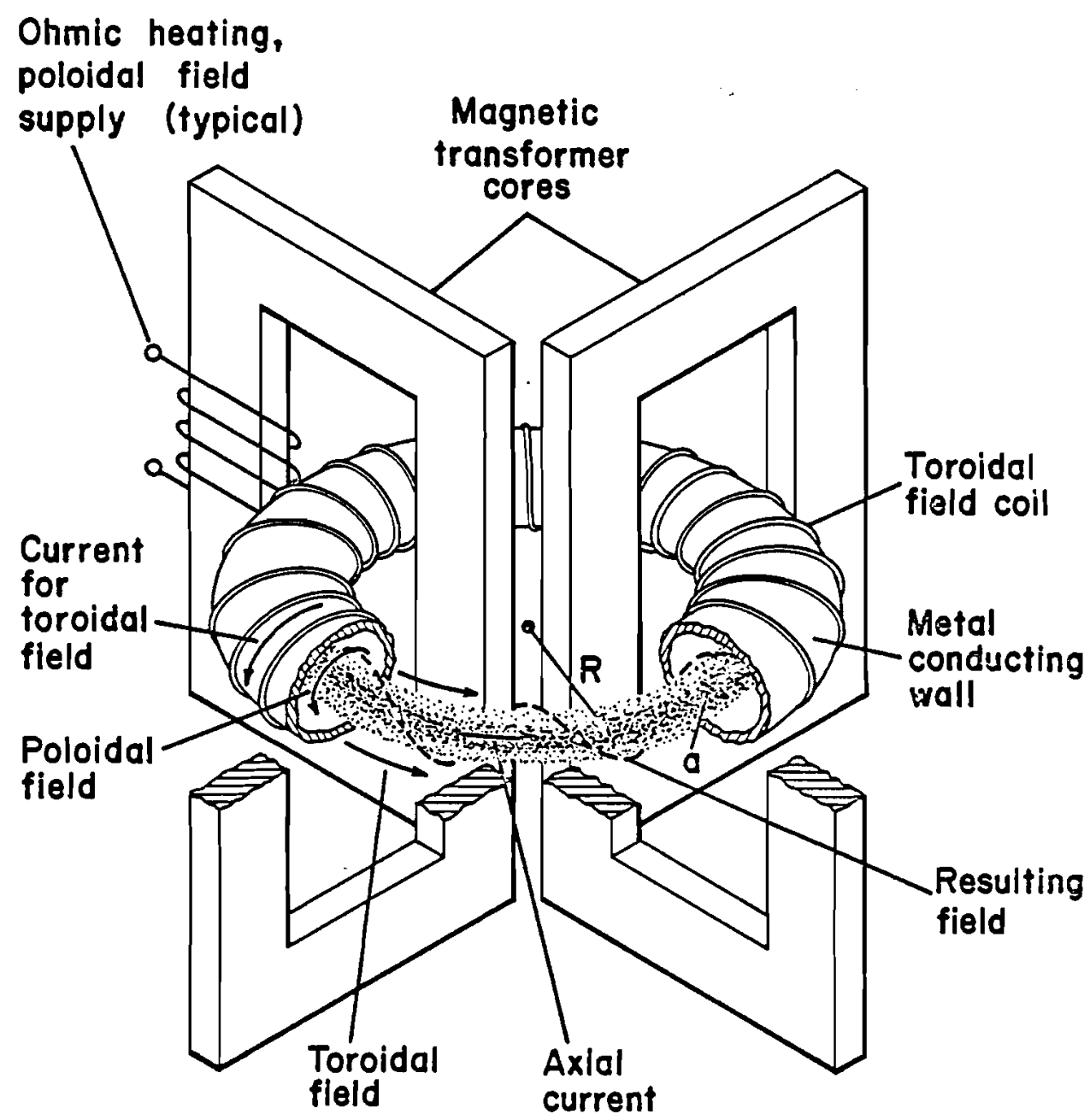

FIGURE 10. Schematic of Tokamak Device

Table 2. Major Tokamak Experiments Which Have Been or Are Now in Operation in the United States

\begin{tabular}{|c|c|c|c|c|}
\hline Experiment & Laboratory & $\begin{array}{l}\text { Major } \\
\text { Radius } \\
(\mathrm{cm}) \\
\end{array}$ & $\begin{array}{l}\text { Minor } \\
\text { Radius } \\
\text { (cm) }\end{array}$ & $\begin{array}{r}\text { Toroidal } \\
\text { Magnetic } \\
\text { Field (kG) }\end{array}$ \\
\hline ST & Princeton & 109 & 14 & 35 \\
\hline ATC & Princeton & 30 & 7 & 60 \\
\hline PLT & Princeton & 130 & 45 & 50 \\
\hline ORMAK & Oak Ridge & 80 & 24 & 25 \\
\hline Doublet II & General Atomic & 59 & $30-90$ & 10 \\
\hline Alcator & M.I.T. & 54 & 12 & 120 \\
\hline$\pi T$ & Univ. of Texas & $: 60$ & 10 & 35 \\
\hline
\end{tabular}




\section{PLASMA INSTABILITIES AND IMPURITY CONTROL}

As the program in controlled thermonuclear fusion got underway in the early 1950s, optimism for success was high. Two important reasons for this optimism were: 1) The motion of single particles in magnetic fields was well understood, and it was postulated that a collection of charged particles in a magnetic field would behave as the sum of its component particles, and 2) Much was known about gaseous discharges, and it was believed the this knowledge would be of significant help.

The situation which developed during the early years of the controlled fusion program was much different from that which had been anticipated:

- Thermonuclear plasmas were found to act quite differently from the behavior predicted for a collection of charged particles. Fusion plasmas were found to be best characterized by collective behavior which had been little studied and which was essentially not understood.

- Gaseous discharge experience was found to be of little help in understanding plasma behavior. Gaseous discharges contain approximately 99 percent neutral gas atoms. Fusion plasmas have less than one percent neutral gas atoms.

Magnetic confinement devices built during the early years of the fusion program were all subject to plasma instabilities which limited confinement times for these machines to values several orders of magnitude below what is required for a fusion reactor. During the second decade of the fusion program, major theoretical and experimental programs were undertaken to identify and understand plasma instabilities and to devise magnetic field configurations capable of stabilizing and confining a plasma for the time necessary to achieve net energy output. A primary reason for the current optimism in the magnetic confinement program is that since the mid-1960s considerable progress has been made in understanding the dynamics of high temperature plasmas. An indication of this is that the results of recent magnetic confinement experiments have tended to confirm predictions based on plasma theory.

A magnetically confined plasma can escape in two ways: as a result of collisions between particles or as a result of instabilities. At the very high temperatures and low densities of fusion plasmas, the collision frequency is so low that the confinement time dictated by collision processes (the so-called classical confinement time) is sufficiently long to meet the Lawson criterion. Plasma instabilities can, however, cause particles and energy to be lost across a magnetic field at much faster rates than are due to particle collisions.

Plasma instabilities can be grouped into two classes: macroscopic and microscopic. Macroscopic instabilities involve large scale motions of the plasma across a confining magnetic field. These instabilities have growth times of the order of microseconds and result in total loss of confinement of the plasma in times which are short compared to classical diffusion loss times. Some macroscopic instabilities are similar to the hydrodynamic instability that develops when a fluid of given density is superimposed on one of lower density in a downward gravitational field. ${ }^{(4)}$ 
Kink instabilities in pinch devices and magnetohydrodynamic instabilities in mirror machines and in low density closed systems (stellarators and tokamaks) are examples of macroscopic instabilitfes. Much has been learned about these instabilities during the past two decades, and magnetic field configurations have been devised which suppress and control them. Kink instabilities can be controlled by eddy currents induced in a conducting shell which surrounds the plasma. Magnetohydrodynamic instabilities are controlled in mirror machines by magnetic wells and in stellarators and tokamaks by helical magnetic fields of the type shown in Figure 9.

Microinstabilities refer to very rapid small scale fluctuations in the density and electric field within a plasma. Whereas macroscopic instabilities can destroy plasma confinement in microseconds, microscopic instability effects generally occur in times of the order of milliseconds and tend to saturate at low level.

Microinstability-induced loss of confinement in magnetic mirror devices arises because wave-particle interactions speed up diffusion-like processes which result in the deflection of plasma particles into the loss cone. Once macroscopic instabilities in mirror systems have been suppressed by use of a magnetic well, deflection of particles into the loss cone destroys the confinement of the plasma. Microinstability losses in mirror machines have been reduced by deliberate control of plasma particle velocity distributions, limitation in the length of the plasma, and use of deep magnetic wells.

Success in controlling instabilities in magnetic mirror devices is shown in Figure 11 which is a plot of plasma density versus time for the $2 X$ facility at Lawrence Livermore Laboratory. The figure shows that if the confinement configuration is a simple magnetic mirror, plasma is lost rapidly to the walls. By using Ioffe bars to produce a magnetic wel1, confinement time is increased as shown in the second curve which was described at the Novosibirsk conference in 1968. A combination of a deep magnetic well and energy spreading of the incident beam to minimize loss cone effects results in the third density decay curve which was obtained in 1970. Spreading the energy of the injected beam still more should make it possible to approach the top curve which represents the classical loss rate for collisional scattering of ions into the loss cone.

In closed systems, the presence of particle and velocity gradients resulting from confinement tends to make plasma particle distributions non-Maxwillian and to cause a relative drift motion between ions and electrons. This drift motion can drive a wave-particle instability. In tokamaks and stellarators, drift instabilities are suppressed by magnetic field configurations which incorporate high shear, net magnetic well and axial symetry. Stellarator field configuration rank relatively lower with regard to these field configurations than do tokamak field configurations. This may account for the improvement in confinement times for tokamak machines over what has been achieved with stellarator devices.

In addition to plasma instabilities, a second probiem preventing the attainment of reactor conditions in early fusion machines was the problem of impurities in the plasma. This problem was especially critical in steady state devices such as the stellarator. Plasma impurities result when material is dislodged from vacuum chamber walls. The dislodged impurity atoms cool the discharge by collision with ions in the plasma. During the early 1960s, two methods for 
control of impurities were investigated. The first method involved the development of "clean" vacuum chamber walls by baking the walls to remove occluded gases. The second method involved use of a device called a divertor. For toroidal machines such as the stellarator or the tokamak, the divertor is a large chamber which encircles the tube of the torus and is connected to the tube by a small opening. By means of special coils, lines of magnetic flux are made to pass through this opening into the divertor chamber. As plasma particles diffuse toward the walls of the torus they are diverted into this chamber where they collide with the chamber wall. The divertor chamber wall has a large surface area which can be cooled and which would be designed to withstand the high energy flux from impinging plasma particles. Extensive research is currently underway on divertor systems for tokamak devices.

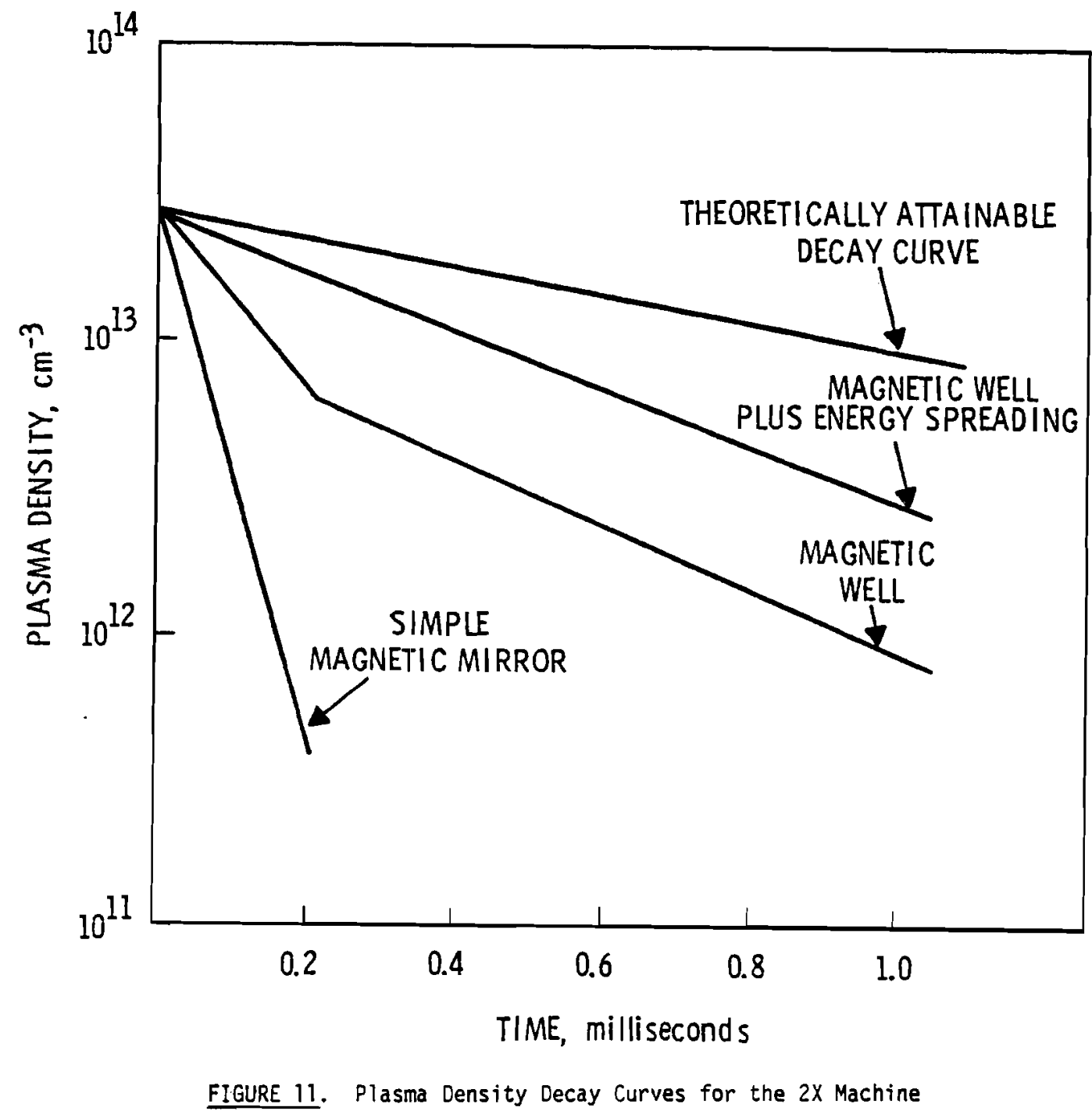




\section{CURRENT STATUS}

Three magnetic confinement concepts: tokamak, theta pinch, and magnetic mirror are currently being investigated by fusion scientists in the United States. Major laboratories where magnetic confinement research is being performed include Princeton Plasma Physics Laboratory (PPPL), Oak Ridge National Laboratory (ORNL), Los Alamos Scientific Laboratory (LASL), Lawrence Livermore Laboratory (LLL), Massachusetts Institute of Technology (MIT) and General Atomic Company. Smaller projects are located at United Technology Research Center, the Naval Research Laboratory, and at several university laboratories. The location and status of the principal magnetic confinement devices in operation or under construction in the United States is shown in Table 3 . The table indicates optimum values of temperature, ion density and confinement time achieved or planned for these devices. Figure 12 shows graphically the progress which is being made toward attainment of temperature and confinement parameters necessary for operation of a DT-burning fusion reactor.

TABLE 3. Principle Magnetic Confinement Devices in Operation or Under Construction in the United States

\begin{tabular}{|c|c|c|c|c|c|c|}
\hline Device & Laboratory & $\begin{array}{c}\text { Date of } \\
\text { Initial } \\
\text { Operation }\end{array}$ & $\begin{array}{c}\text { Confinement } \\
\text { Time } \\
\text { (sec) } \\
\end{array}$ & $\begin{array}{l}\text { Particle } \\
\text { Density } \\
\text { (cm-3) }\end{array}$ & $\left(\mathrm{cm}^{n \pi} \mathrm{sec}\right)$ & $\begin{array}{c}\text { Ion } \\
\text { Temperature } \\
\text { (keV) }\end{array}$ \\
\hline \multicolumn{7}{|l|}{ Tokamak } \\
\hline ST & PPPL & 1970 & $20 \times 10^{-3}$ & $3 \times 10^{13}$ & $6 \times 10^{11}$ & 0.5 \\
\hline ORMAK & ORNL & 1971 & $10 \times 10^{-3}$ & $3 \times 10^{13}$ & $3 \times 10^{11}$ & 0.4 \\
\hline ATC & PPPL & 1972 & $5 \times 10^{-3}$ & $10^{14}$ & $5 \times 10^{11}$ & 0.7 \\
\hline Doublet II & Gen. Atomics & 1971 & $20 \times 10^{-3}$ & & & \\
\hline Alcator & MIT & 1975 & $20 \times 10^{-3}$ & $5 \times 10^{14}$ & $1 \times 10^{13}$ & 0.7 \\
\hline PLT & PPPL & 1975 & $50 \times 10^{-3}$ & $10^{14}$ & $5 \times 10^{12}$ & 2.0 \\
\hline PDX & PPPL & $1978 *$ & & & & \\
\hline Doublet III & Gen. Atomics & $1978 *$ & & & $10^{14 *}$ & $5.0^{*}$ \\
\hline Alcator C & MIT & $1978^{*}$ & $0.1^{*}$ & $10^{15^{*}}$ & $1 \times 10^{14^{*}}$ & $2.0^{*}$ \\
\hline ORMAK Upgrade & ORNL & $1978^{*}$ & $30 \times 10^{-3 *}$ & $10^{14 *}$ & $3 \times 10^{12 *}$ & $3.0^{*}$ \\
\hline TFTR & PPPL & $1981 *$ & $0.5^{*}$ & $10^{14 *}$ & $5 \times 10^{13 *}$ & $5.0^{*}$ \\
\hline
\end{tabular}

High Oensity Systems

$\begin{array}{llccccc}\text { SCYLLAC } & \text { LASL } & 1974 & 3 \times 10^{-5} & 3 \times 10^{16} & 9 \times 10^{11} & 1.0 \\ \text { STP } & \text { LASL } & 1975 & 2.5 \times 10^{-5} & 3 \times 10^{15} & 7.5 \times 10^{10} & 1.2 \\ 2 T-S & \text { LASL } & 1975 & 1.5 \times 10^{-5} & 10^{15} & 1.5 \times 10^{10} & 0.15 \\ \text { SCYLLA-IY-P } & \text { LASL } & 1976 & 2 \times 10^{-5} & 3 \times 10^{16} & 6 \times 10^{11} & 3.0 \\ 2 T-40 & \text { LASL } & 1978^{*} & 10^{-4 *} & 10^{*} & 10^{11 *} & 0.3^{*}\end{array}$

Magnetic Mirror

\begin{tabular}{|c|c|c|c|c|c|c|}
\hline Baseball-II & LUL & & 0.7 & $4 \times 10^{9}$ & $3 \times 10^{9}$ & 1.0 \\
\hline $2 x$ & LLL & & $10^{-3}$ & $110^{13}$ & $10^{10}$ & 8.0 \\
\hline $2 x-11$ & LLL & & $0.5 \times 10^{-3}$ & $5 \times 10^{13}$ & $2.5 \times 10^{10}$ & 3.0 \\
\hline LITE & UTRC & 1974 & $5 \times 10^{-6}$ & $10^{13}$ & $5 \times 10^{7}$ & 1.0 \\
\hline Baseball-II T & ULL & $1976^{*}$ & $5 \times 10^{-3 *}$ & $>10^{13 *}$ & $210^{11 *}$ & $10-15^{*}$ \\
\hline $2 x-11 \quad B$ & LLL & 1975 & $>5 \times 10^{-3}$ & $4 \times 10^{13}$ & $2 \times 10^{11}$ & 13 \\
\hline MX & LLL & $1987^{*}$ & $>10^{-2 *}$ & $10^{14 \star}$ & $10^{12 *}$ & $50 \star$ \\
\hline
\end{tabular}

*Anticipated completion dates and expected parameters for devices under construction. 


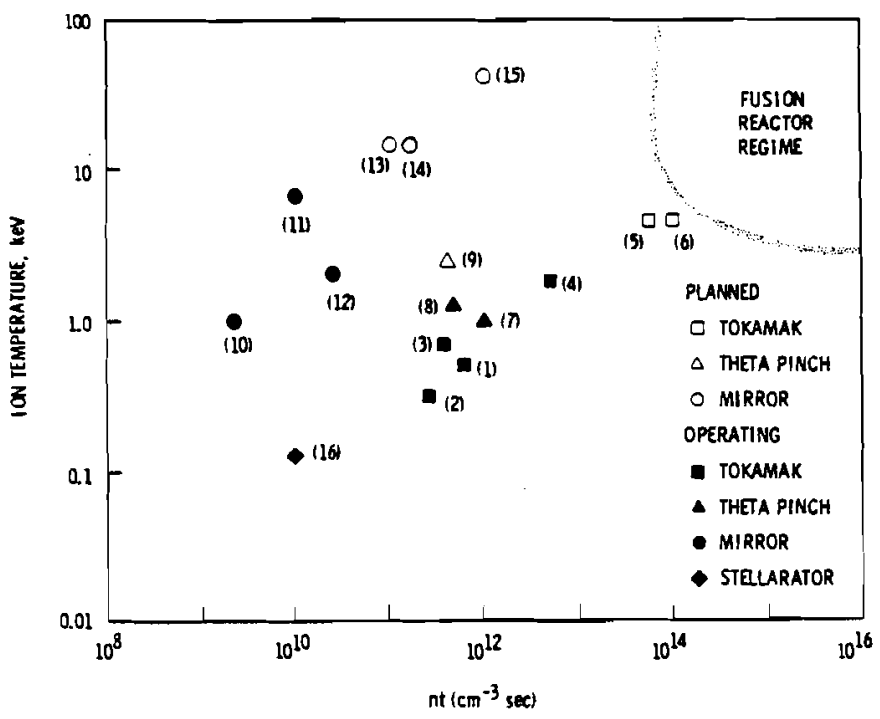
(1) ST Tokamak
(2) ORNAK
(3) ATC
(4) PLT
(5) TFTR
(6) Doublet IIt
(7) SCYLLAC
(8) STP
(9) SCYLLA-IV-P
(10) Basebal1-II
(II) $2 x$
(12) $2 x-15$
(13) Basebal1-11 T
(14) $2 x-11 B$
(15) $\mathrm{MX}$
(16) Model-c Stellarator

FIGURE 12. Progress in Attainment of Fusion Reactor Conditions

Accomplishments of operating magnetic confinement devices and objectives of devices currently under construction $(12,13,14,15)$ are summarized below.

Tokamak

The United States program in tokamak physics began in 1968 and included conversion of the Model C Stellarator at Princeton to the ST (Symmetric Tokamak) device and construction of ORMAK at Oak Ridge National Laboratory. Soviet tokamak successes were confirmed in the ST in 1970-1971. ORMAK began operation in 1971, and by February 1973 had provided the first test of tokamak scaling laws* at larger plasma size. Comparison of the performance of ST at PPPL and ORMAK at ORNL showed results that are consistent with theoretical scaling laws for plasma size and magnetic field strength.

Initial heating of tokamak plasmas is accomplished by an electric current flowing through the plasma (ohmic heating). Ohmic heating depends on the electrical resistance of the plasma, much like a conventional resistance heater. At temperatures above about one million degrees Kelvin, the resistance of the plasma decreases to the point where ohmic heating ceases to be useful. To achieve fusion temperatures, it is necessary to provide supplemental heating. Methods currently under study to heat a plasma include compressional heating due to a sudden increase in the confining magnetic field, radio frequency ( $r f$ ) heating, injection into the plasma of a beam of high energy neutral atoms, and the use of laser beams.

An experiment on compressional heating was started at PPPL on Adiabatic Toroidal Compressor (ATC) in 1972. The experiment achieved an increase in ion temperature by a factor of 3 with an ion density of approximately $10^{14} \mathrm{~cm}^{-3}$. The size of the device was chosen to demonstrate the heating method rather than to achieve reactor conditions, and it performed according to theory.

\footnotetext{
ॠScaling in fusion systems refers to the physical laws wrich relate plasma confinement time to device size, magnetic field strength, plasma temperature, etc. The determination of scaling laws by theory and experiment is a key factor in establishing the economics of fusion reactors.
} 
Heating of tokamak plasma by means of high energy neutral beam injection has also been successfully demonstrated with both ATC and ORMAK. Experiments with ORMAK have given the following results: with $90 \mathrm{kw}$ of heating power injected into a plasma carrying $155 \mathrm{kA}$ of plasma current the ion temperatures were $325 \mathrm{eV}$ with ohmic heating; $430 \mathrm{eV}$ with ohmic heating and cotnjected neutral beams; and $380 \mathrm{eV}$ with ohmic heating and counter-injected neutral beams.

ORMAK can be modified to operate at higher magnetic fields than the 25 kilogauss now employed. An increase in plasma current, made possible by an increased magnetic field, and an increase in neutral beam injection power has yielded ion temperatures in the keV range. Modification of ORMAK to increase the toroidal field strength and the ion current is presently underway. To further explore neutral beam heating, high powered neutral beams ( $22 \mathrm{MW}$ ) will be injected into the ORMAK UPGRADE with the expectation of doubling the ion temperature.

In tokamak devices the confinement time and plasma temperature are predicted by theory to increase strongly with increased physical size (i.e., increased plasma volume). A device which will provide important experimental information about tokamak scaling laws has recently been completed at Princeton Plasma Physics Laboratory. The first plasma in Princeton Large Torus (PLT) was achieved in December 1975. Design parameters for PLT are such that the simultaneous conditions of high density, high plasma temperature, and long energy confinement times should be approached for the first time in a large volume tokamak with hydrogen plasma. Initial results indicate the expected increase of the energy containment time with size. Neutral beam heating will supplement ohmic heating in PLT in 1977. This supplemental heating is expected to make possible the attainment of ion temperatures in the 2 to $5 \mathrm{keV}$ range.

A technique for lowering the magnetic field strength required to confine a tokamak plasma by making the plasma cross-section noncircular has been demonstrated by the Doublet II device at General Atomic Company. The importance of the Doublet experiments with a kidney shaped plasma is that they point to the possibility of achieving large plasma volumes with smaller magnetic fields than are theoretically possible with tokamak plasmas of circulgr cross section. Success of the Doublet experiments could therefore have important economic consequences. Doublet III, a large noncircular cross section hydrogen plasma device which is expected to achieve temperature and confinement parameters close to the fusion reactor regime is scheduled to be operational in early 1978.

The Alcator II device at MIT is exploring plasma confinement in high magnetic fields. The value of $\mathrm{nt}$ has been increased to $1.0 \times 10^{13} \mathrm{~cm}^{-3} \mathrm{sec}$ and the plasma is extremely clean. A new high magnetic field device, Alcator $C$, is under construction and is expected to produce an $n \tau$ of approximately $1.0 \times 10^{14} \mathrm{~cm}^{-3} \mathrm{sec}$ with a piasma temperature of $2 \mathrm{keV}$.

One of the major problems with tokamak machines is the buildup of impurities in the plasma. Poloidal Divertor Experiment (POX), a large hydrogen device under construction at PPPL, is designed to test the use of magnetic divertors to reduce plasma-wall interactions and maintain impurity concentrations at manageable levels. PDX is scheduled for operation in early 1978. 


\section{High Density Sys tems}

Experiments with Scylla and Scyllac devices at Los Alamos have demonstrated the possibility of achieving fusion reactor conditions in pulsed, theta-pinch devices. The Scylla IV linear theta pinch experiment achieved plasma densities of approximately $3 \times 10^{16} \mathrm{~cm}^{-3}$ and ion temperatures of about $4 \mathrm{keV}$ ( $45 \mathrm{million}$ degrees Kelvin). The Scylla IV-P device began operating in 1976 and is a $5 \mathrm{~m} l$ linear theta pinch designed to study end-loss and end-stoppering of linear devices. The parameters of this device are similar to the earlier Scylla IV experiment.

Experiments with 5- and 8-meter toroidal sectors (Figure 13) demonstrated toroidal equilibrium which agrees with theoretical predictions. Experiments with full torus Scyllac, which began in 1974, demonstrated the presence of $m=1$ instability. In the present derated sector of this torus feedback stabilization of the $m=1$ instability is being investigated. Confinement time has been increased to about 30 microseconds. The goal of Scyllac experiments is confinement for $50-100$ microseconds corresponding to nt values of 2 to $5 \times 10^{12} \mathrm{~cm}^{-3} \mathrm{sec}$.

Plasma heating in theta pinch devices is accomplished in two stages: shock heating followed by compressional heating. Scylla and Scyllac devices operated to date have employed a single capacitor bank power supply for both stages. Future devices designed to achieve reactor operating conditions will require separation of the energy sources for the two stages. Separation of the energy sources is necessary for economic considerations as well as the need to produce greater implosion heating and utilize less adiabatic compression for improved stabilization of the plasma.

Construction of a Staged Theta Pinch (STP) machine to demonstrate the use of separate energy sources for plasma heating in theta pinch reactors was completed at LASL in 1975. The system utilizes a low-energy high-voltage capacitor bank for fast shock heating of the plasma and a high-energy magnetic storage system with a superconducting storage magnet for final compression. A staging capacitor bank, similar to the present Scyllac bank, bridges the time scale between shock heating and compression. Experiments with the Staged Theta Pinch are expected to yield ion temperatures of about $1.5 \mathrm{keV}$ at plasma densities of $7 \times 10^{15} \mathrm{~cm}^{-3}$.

Another high density concept is the toroidal Z-pinch which is also known as Reversed Field Pinch (RFP). Z-pinch experiments are being carried out at LASL in the United States and at fusion laboratories in England, Italy and Japan. The RFP is a toroidal device, generically similar to a tokamak, but with a smaller toroidal field strength to plasma current ratio and a larger toroidal diameter to plasma cross section diameter ratio than exists for tokamak. Present RFP research at LASL is being carried out on the $Z T-S$ device. A larger device, $Z T-40$ is in the design stage. 


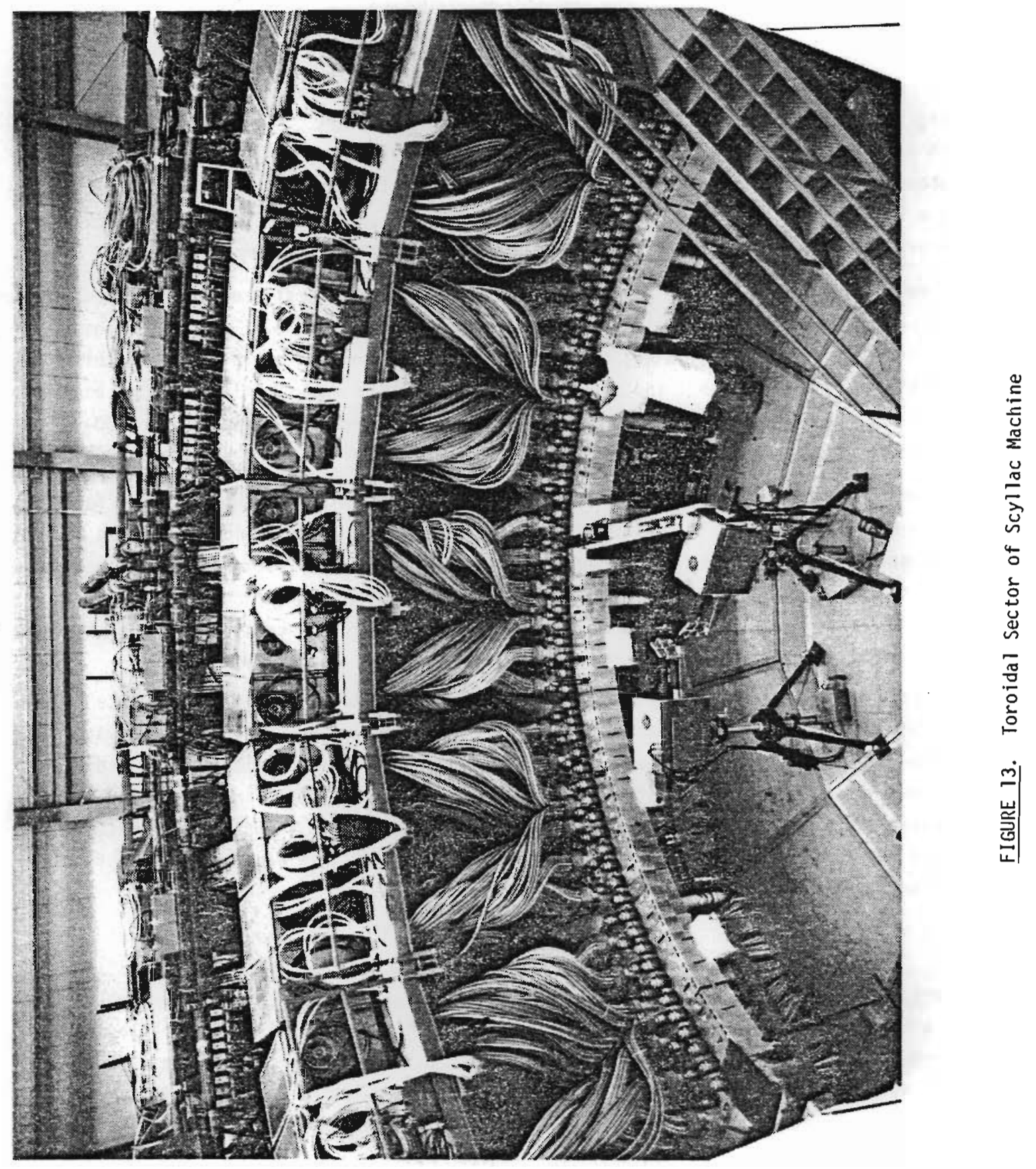




\section{Magnetic Mirrors}

The stability and scaling with energy and size of classical mirror devices are being investigated in magnetic mirror experiments at Lawrence Livermore Laboratory (LLL). Methods are being investigated to improve the $Q$ of a mirror reactor $(Q=$ fusion power out/beam power in). Present calculations indicate that classical mirror reactors have $Q \simeq 1$. In a mirror machine the high temperature plasma is produced and maintained against leakage through the mirrors by a continuous input of energetic neutral beam atoms. To suppress MHD instabilities, present day machines use magnets that generate fields having a minimum point such that the magnitude of the field increases in every direction away from the center (a magnetic well). Mirror machines are relatively free of impurity atom contamination problems because impurities tend to be expelled out the ends of the machine as fast as they enter the plasma.

The major experiment in the present mirror program is the $2 X-I I B$, located at LLL. This device (Figure 14) employs a compression magnet consisting of two C-shaped coils (Yin Yang coils) oriented at right angles and centered about a common point. The hot plasma is obtained by neutral beam injection onto a warm plasma stream target. The present facility uses twelve, 20 keV, $50 \mathrm{Amp}$ neutral beam sources to inject up to 300 Amp of deuterium atoms. This injection plus the additional stabilization given by a warm plasma stream results in a hot $(9-13 \mathrm{keV})$, dense $10^{13}-10^{14} \mathrm{~cm}^{-3}$ ) plasma, with an $\mathrm{nt}$ of about $10^{11} \mathrm{~cm}^{-3} \mathrm{sec}$. Additional planned modifications will increase the beam injection energy from $20 \mathrm{keV}$ to $40 \mathrm{keV}$ to test the plasma stability and energy scaling at iigher ion temperatures.

Baseball IIT is designed to study stable confinement of hot, dense plasmas under steady-state conditions. Experiments are being conducted with a laser produced target plasma for initial startup, with additional plasma heating supplied by neutral beam injection.

The use of laser beams to produce the initial plasma in mirror machines is also being investigated in the Laser Initiated Target Experiment (LITE), a sma11 mirror experiment at the United Technologies Research Center in East Hartford, Connecticut. The target in the LITE experiment is a solid LiH particle which is vaporized by two-sided irradiation from a pulsed Nd-glass laser. Neutral beam injection is then used for additional heating.

MX (Figure 15) is a proposed large mirror experiment which is scheduled to begain operation in 1981. MX will extend the physics experiments currently underway on 2X-IIB and Baseball IIT to much larger sizes and higher energies. MX will utilize a large superconducting magnet for steady state operation and is expected to produce near-reactor conditions for the first time in a mirror system. 


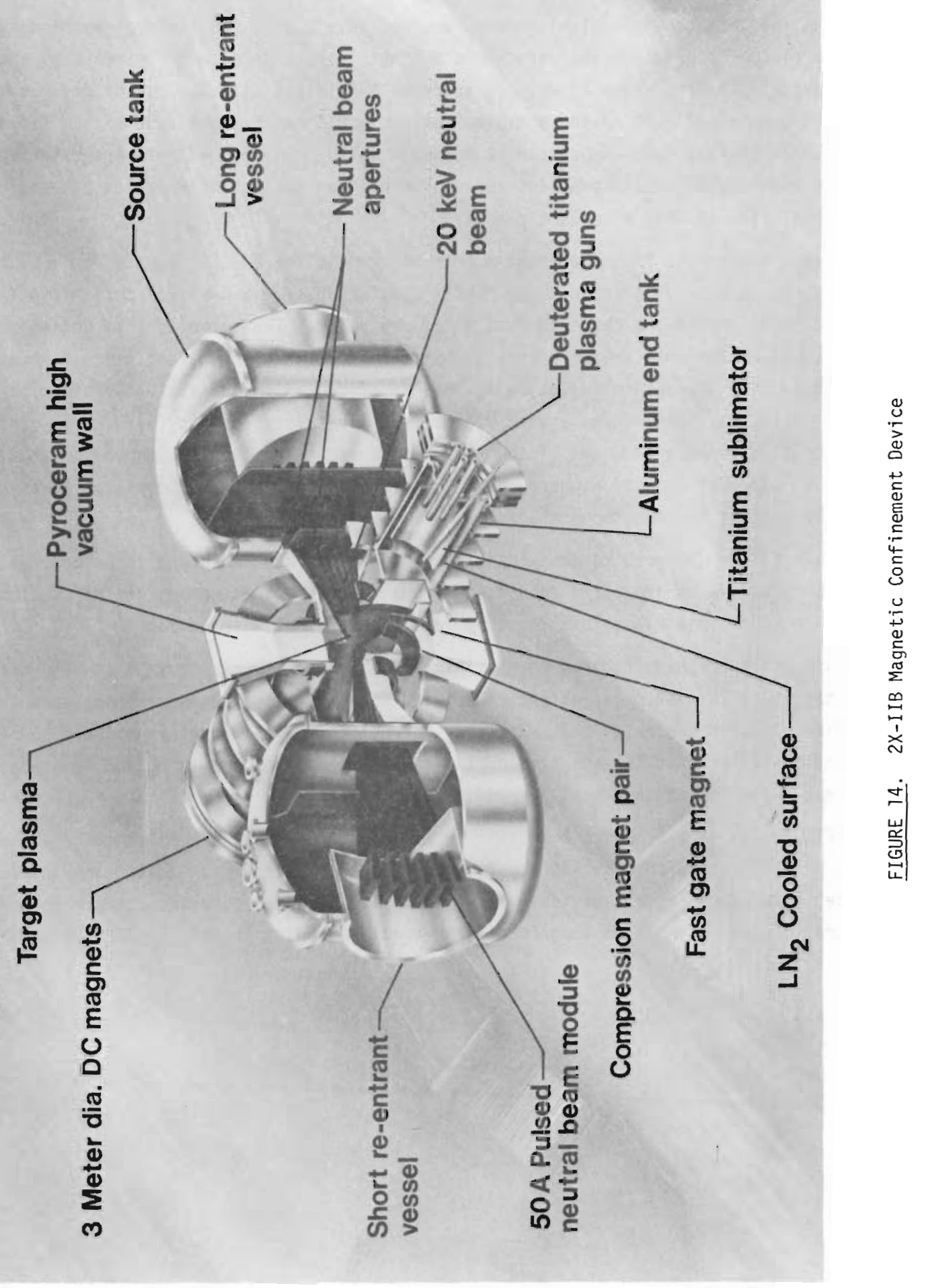




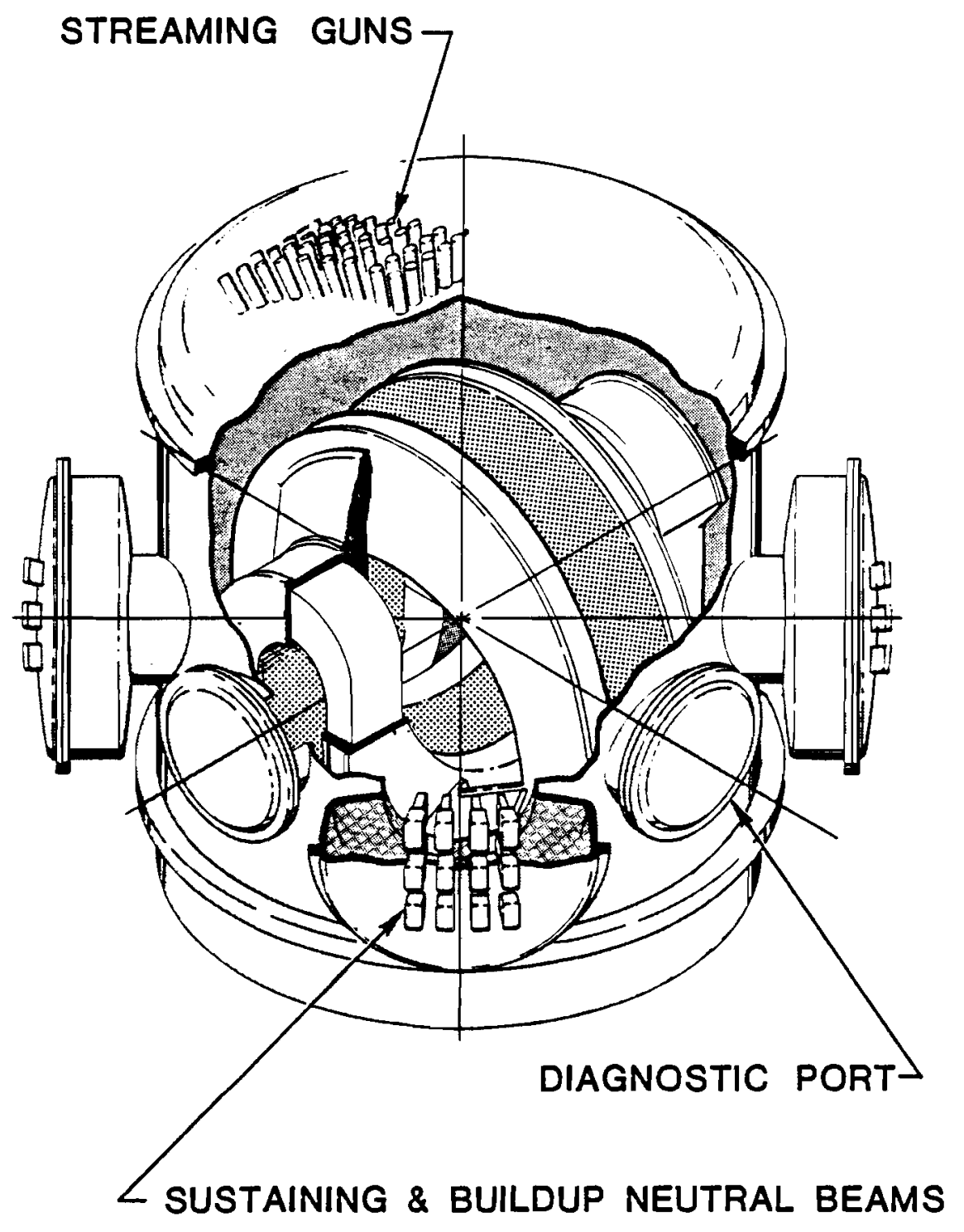

FIGURE 15. Proposed MX Facility 


\section{INERTIAL CONFINEMENT RESEARCH}

Inertial confinement represents an al ternative to the process of magnetic confinement of a fusion plasma. The basic idea of inertial confinement is to compress and heat a tiny fuel pellet with the result that fusion reactions occur during the short period of time, set by the inertia of the system, before particles within the system fly apart and terminate the reaction. For a deuterium-tritium fuel pellet, net energy production will occur from fusion reactions if the product of plasma density and confinement time is of the order of $10^{14} \mathrm{~cm}^{-3} \mathrm{sec}$ (the Lawson criterion). With inertial confinement times of about $10^{-12} \mathrm{sec}$, the required plasma densities are $10^{26} \mathrm{~cm}^{-3}$. Thus, the fue 1 must be compressed to about 10,000 times its normal 1 iquid density. Both high-power short-duration laser pulses and strongly focused beams of high energy electrons are being considered as the agent for compression and heating of the fuel pellet.

As with magnetic confinement systems, the critical issue for inertial confinement systems is stability. In order for pellet fusion to succeed, the laser (or electron beam) must rapidly and symmetricaliy compress and heat the fuel pellet. Any process which results in asymmetric compresion or in premature heating of the pellet interior will prevent achievement of the high pellet density required by the Lawson criterion as a condition for net energy production.

To date, the major effort in inertial confinement systems has invoived the use of laser beams for pellet compression (laser fusion). Several review articles which describe the laser-fusion process have appeared in the literature. (17-21) A summary of the current status of the United States laser-fusion program has also been published. (22)

Basic work on the use of inertial confinement for the production of very large thermonuclear reactions for weapons applications was carried out during the forties and fifties at laboratories in the United States and abroad which were engaged in the development of the hydrogen bomb. The idea of using a spherical implosion to produce the high compression necessary for a fusion reaction was developed by scientists at the Los Alamos Scientific Laboratory during the early 1940s. With the conclusion of the Second World War, attention turned to peaceful applications of nuclear energy. The physical principles of controlled fusion microexplosions and some of the techniques for realizing these principles were defined by E. Teller and S. Ulam in an internal technical document written at Los Alamos in 1951.

Initialiy, use of the inertial confinement concept for controlled fusion microexplosions was thought to be impractical because of the lack of a suitable implosion mechanism. Shortly after the invention of the pulsed ruby laser in 1960, it was recognized that this device could, in principle, provide the concentration of energy needed to compress and heat a plasma to the point where fusion reactions would occur. During 1960 and 1961, scientists at Los Alamos and at Livermore performed calculations which showed that a spherical implosion might be produced by a time-tailored laser pulse, and that very high compression of the pellet material could result. However, very high energy pulses would be required, and at the time of these initial calculations the status of laser technology was such that only relatively low laser energies were available.

The decade following the invention of the laser in 1960 was a period of rapid development in laser technology. Invention of the Q-switching technique in 1962 led to creation of the 
giant-pulse laser with an energy of the order of 1 Joule per pulse. Invention of the modelocked laser in 1964 permitted the generation of nanosecond and later of picosecond pulses. These developments led to the construction of large oscillator-amplifier chains which today are capable of delivering hundreds of Joules of energy in a single sub-nanosecond pulse.

In 1969, programs were initiated at Livermore and at Los Alamos to reevaluate the laserpellet approach to controlled thermonuclear fusion. Detailed calculations based on computergenerated models revealed that with optimal pulse shaping the laser energy required to trigger a fusion microexplosion in a solid OT pellet could be reduced by about three orders of magnitude from earlier estimates to a value of $10^{5}$ or $10^{6}$ Joules which appeared to be within reach of foreseeable laser technology. The interest in laser fusion resulting from these calculations has led to significantly expanded activity in the areas of laser development, D-T target development, optical delivery systems development, and modeling studies of the laser-fusion process.

Because of the use of military technology, laser-fusion research was, until recently, strictly classified, and some of the research remains in that category. Very little theoretical work on laser fusion was published during the 1960s. The first published papers indicating that lasers could be used to produce plasmas of thermonuclear interest appeared in 1964 and included publications by Dawson ${ }^{(23)}$ in the United States and by Basov and Krokhin (24) in the USSR. Information about the implosion concept, including details about laser energy requirements, was released to the public in 1971. Information about the physical properties of the D-T fuel pellet was declassified in 1974 .

Government sponsored research in laser fusion is centered at three laboratories: Lawrence Livermore Laboratory (LLL), Los Alamos Scientific Laboratory (LASL), and Sandia Laboratory (SLA). A smaller laser fusion research program is also located at the Naval Research Laboratory. Significant privately funded programs have been developed in recent years at the Battelle Memorial Institute, Columbus, Ohio, at KMS Fusion, Inc.: Ann Arbor, Michigan, and at the University of Rochester, New York.

Current research on laser fusion is aimed at demonstrating the feasibility of the laserpellet inertial confinement concept as a viable alternative to controlled fusion based on magnetic confinement. The feasibility demonstration involves severai steps: 1) the development of suitable high peak-power short-pulse lasers together with the necessary hardware for delivery of the laser energy to the fuel pellet; 2) design and fabrication of O-T target pellets; 3) continuation of the large scale computer calculations on both the laser-plasma interaction and on pellet compression in order to investigate the complex physical processes that occur; 4) an actual experimental demonstration of laser induced compression and thermonuclear burn of a fusion pellet.

Present laser-fusion experiments are being conducted with neodymium-glass (Nd-glass) and $\mathrm{CO}_{2}$-gas laser systems. It is believed that these systems can be scaled to energy leavels sufficient to demonstrate significant thermonuclear burn (TN-burn) and even scientific break-even (fusion energy output equal to laser energy input). However, systems employing an entirely new laser concept will be required to provide the energy for pellet compressions that will result in substantial energy gain. 
A summary of existing $\mathrm{Nd}-\mathrm{glass}$ and $\mathrm{CO}_{2}$-gas laser systems at laboratories where government

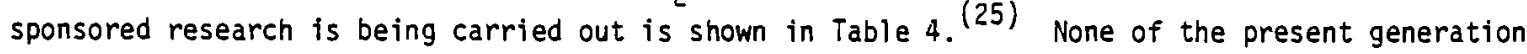
of lasers has proved adequate to demonstrate scientific breakeven, al though neutron production from fusion has been observed in some experiments. (26)

Efforts to develop very large laser systems are currently underway at the natonal laboratories. These include Lawrence Livermore Laboratory where a 10,000 Joule $\mathrm{Nd}-\mathrm{glass}$ laser system is under construction and Los Alamos Scientific Laboratory where a 100,000 Joule $\mathrm{CO}_{2}$-gas laser is being designed (see section on Fusion Program Development). The Nd-glass program at Sandia Laboratories is being de-emphasized in favor of development of electron-beam initiated chemical lasers and electron-beam fusion studies.

To provide theoretical guidance for the laser fusion program, numerical computer codes which model the laser-fusion implosion process in two dimensions are in operation at both Lawrence Livermore Laboratory and Los Alamos Scientific Laboratory. These codes are used to model the interaction of incident laser energy with deuterium-tritium targets and to chart the course of an implosion-produced microexplosion. The codes provide necessary guidance for the design of suitable lasers and target systems.

Table 4. Existing Laser Systems at Government-Sponsored Reserach Laboratories

\begin{tabular}{|c|c|c|c|c|}
\hline Laser & Type & Energy/Time & Location & $\begin{array}{c}\text { Year } \\
\text { Operational } \\
\end{array}$ \\
\hline Nd-glass & I beam & $100 \mathrm{~J} / 1 \mathrm{nsec}$ & LLL & 1969 \\
\hline Nd-glass & 4 beam & $300 \mathrm{~J} / 1 \mathrm{nsec}$ & SLA & 1974 \\
\hline Nd-glass & 4 beam & $500 \mathrm{~J} / 0.35 \mathrm{nsec}$ & LASL & 1974 \\
\hline Nd-glass & 2 beam & $150 \mathrm{~J} / 0.1 \mathrm{nsec}$ & LLL & 1974 \\
\hline $\mathrm{Nd}$-glass & $8-12$ beam & $1000 \mathrm{~J} / 0.15 \mathrm{nsec}$ & LLL & 1975 \\
\hline $\mathrm{CO}_{2}$-gas & 1 beam & $250 \mathrm{~J} / \mathrm{I} \mathrm{nsec}$ & LASL & 1973 \\
\hline $\mathrm{CO}_{2}$-gas & 1 beam & $50-100 \mathrm{~J} / 1$ nsec & LLL & 1974 \\
\hline $\mathrm{CO}_{2}$-gas & 1 beam & $2500 \mathrm{~J} / 1 \mathrm{nsec}$ & LASL & 1975 \\
\hline
\end{tabular}

KMS Industries and the U.S. Atomic Energy Commission entered into a no-cost contract on February 3,1971 that allows KMS Fusion to develop Iaser fusion technology for peaceful purposes and provides control of the classified information generated in the work. KMS Fusion has completed a sophisticated two-beam target irradiation facility using a neodymium-glass laser with an output of approximately 200 Joule with subnanosecond pulse widths. With this system, KMS has reported implosion generated fusion neutron yields in the range of $10^{5}$ to $10^{6}$ neutrons for a series of D-T pellet irradiation experiments. (22)

A 12-beam Nd-glass laser system has been developed at Battelle Columbus Laboratories which uses beam splitting optics in conjunction with six neodymium-glass laser amplifiers. Each beam in the 12-beam system delivers approximately 120 Joules in 3-1/2 nanoseconds. The 12 pulses have a total energy of 1500 Joules and can be directed onto a target so that they strike the target simultaneously. (27) One significant result from the Battelle program is the demonstration 
that a short pulse laser with energy greater than a kilojoule can be constructed and used for plasma studies without damage to the system from light backscattered from the target, and without causing premature destruction of the target from super-radiance. A second point of significance for laser fusion is that in experiments with metallic targets large conversion efficiencies of laser light to $x$-rays were obtained in accordance with theoretical predictions.

The Laboratory for Laser Energetics at the University of Rochester has constructed a 4-beam $\mathrm{Nd}$-glass laser system that has delivered 400 Joules on target in 100 picoseconds and 1200 Joules in 500 picoseconds. The system has been engineered to a point where the four beams coincide to within approximately \pm 7 picoseconds. A pulse stacker system has also been developed which allows pulses to be time-tailored for use in pellet compression experiments. A recent agreement between the University of Rochester, the Energy Research and Development Administration, and several other private and public sponsors ${ }^{(28)}$ will permit expansion of the Laboratory for Laser Energetics to accormodate a 10 kilojoule neodymium glass laser system being planned for use in laser-fusion experiments.

The possibility of using intense beams of relativistic electrons to provide the energy for implosion and heating of D-T pellets to fusion conditions is being investigated in experimental and theoretical programs at Sandia Laboratories. (29) Preliminary calculations(30) indicate that electron energies of 1 to $10 \mathrm{MeV}$, and current densities of $10^{7}$ to $10^{8}$ amperes $/ \mathrm{cm}^{2}$ would be required with pulses 1 imited to a time duration of 1 to 10 nanoseconds.

Electron beam generators were originally developed by the military for weapons simulation studies. Therefore much of the research on relativistic electron beams is classified, and details of electron beam-fusion studies are just beginning to be available. Experiments performed at Sandia in 1974 showed that relativistic electrons behave as predicted when they impinge on high density solid targets. ${ }^{(31)}$ Compression of hemispherical gold shells by highenergy electron beams has been observed. (32)

The design of accelerators to produce relativistic electron beams with characteristics suitable for electron beam-fusion experiments, and methods of efficiently transmitting and focusing a relativistic beam on a target are presently under study at Sandia Laboratories. 


\section{FOREIGN PROGRAMS}

Research toward the development of controlled nuclear fusion as a source of power began independentiy and almost simultaneously in the United States, Great Britain and the USSR in the early 1950s. For almost a decade, this research was carried out under provisions of utmost secrecy. Information that fusion programs were underway was publicly disclosed in August 1955 at the First International Conference on Atomic Energy held at Geneva, Switzerland; however, no details of the research were given at that time.

At the Second International Conference on Atomic Energy in 1958, fusion exhibits and papers on fusion concepts and problems were presented by scientists from the United States, the United Kingdom and the USSR. This conference can be said to mark the removal of secrecy from fusion studies and the initiation of an era of cooperation in the field of controlled thermonuclear research.

Since 1958 a number of nations have launched fusion programs. Table 5 lists names and locations of major foreign fusion laboratories. Descriptions of worldwide facilities for controlled fusion research can be found in a recent publication of the International Atomic Energy Agency. (33) Seventeen countries (including the United States) are represented in this publication.

As of 1976 the fusion efforts of the United States and the USSR each represent about one-third of the total world effort measured in man-years of effort. Significant new fusion research programs are being carried out in France, West Germany, Italy and Japan. The British effort has declined and is now about equal to that of either France or West Germany. The Japanese effort has grown rapidly and currently represents about 10 percent of the world effort.

Table 5. Major Foreign Fusion Laboratories

Country
France
France
Germany, Fed. Rep.
Germany, Fed. Rep.
Italy
Japan
Netherlands
United Kingdom
USSR
USSR
USSR
USSR
USSR
USSR

Laboratory
Centre d'Etudes Nucléaires
Centre d'Etudes Nucleaires
Max Planck Institute fur
Plasmaphysik
Institute fur Plasmaphysik der
Kernforschingsangler
Laboratorie Gas Ionizzati
Tokai Research Establishment, JAERI
FOM-Instituit voor Plasmaphysica
UKAEA Culham Laboratory
Ioffe Physico-Technical Institute
Physico-Technical Institute
Kurchatov Institute of Atomic Energy
Lebedev Institute of Physics
Physico-Technical Institute
Nuclear Physics Institute

Location
Fontenay-aux-Roses
Grenoble
Garching
Jülich
Frascati
Tokai-mura
Jutphaas
Abingdon, Berkshire
Leningrad
Kharkov
Moscow
Moscow
Sukkumi
Novosibirsk


The largest foreign CTR program is that of the Soviet Union. The major Soviet fusion research laboratory is at the Kurchatov Institute near Moscow, (34) but fusion research is also carried out at five other Soviet research centers (see Table 4). The USSR has about 50 plasma physics experiments and fusion devices in operation. Magnetic confinement devices being studied include the tokamak, plasma-focus (continuous flow pinch), magnetic mirror, and stellarator devices. A significant laser-fusion program is also underway. The Soviets also have an outstanding theory program.

A major breakthrough in magnetic confinement systems technology was announced by the Soviets in 1968 at the Third IAEA Conference on Plasma Physics and Controlled Nuclear Fusion Research. The breakthrough involved the tokamak, a toroidal device which utilized a large circulating current, induced in the plasma by transformer action, to stabilize the plasma. Tokamak devices have achieved significantly larger $n \tau$ values than stellarator machines which employ external helical coils for plasma stabilization. Tokamak operating parameters announced by the Soviets in 1968 for the T-3 device included a hydrogen plasma ion temperature of $0.5 \mathrm{keV}$, a plasma density of $5 \times 10^{13}$ ions $\mathrm{cm}^{-3}$, and a confinement time of $210 \mathrm{milliseconds}\left(\mathrm{n} \tau=5 \times 10^{11}\right.$ ions cm $\mathrm{cm}^{-3} \mathrm{sec}$. Similar results have been achieved by the Russians with a deuterium plasma in tokamak T-4.

The newest and largest Soviet tokamak, T-10, is comparable in size and magnetic field to the PLT at Princeton University. Design parameters include a major radius of $150 \mathrm{~cm}, \mathrm{plasma}$ minor radius of $35 \mathrm{~cm}$, and toroidal magnetic field of $50 \mathrm{kG}$. T-10 produced its first plasma in June 1975. Recent results ${ }^{(35)}$ reported for $T-10$ operation at a toroidal field of $35 \mathrm{kG}$ include: plasma density of $5 \times 10^{13} \mathrm{~cm}^{-3}$, ion temperature of $0.6 \mathrm{keV}$, and confinement time of 50 to $60 \mathrm{msec}$. These preliminary results are most encouraging because they represent an increase over previous typical tokamak values of 3 to 4 in confinement time.

Collaboration between the United States and the USSR has begun to play an important role in the development of fusion power. At a meeting in June 1973 between the heads of state of the two countries, agreement was reached to permit scientific and technical cooperation by researchers working on problems of conversion of the energy of fusion to electrical energy. (36) This cooperation is supervised by a sixteen member group called the Joint (US-USSR) Fusion Power Coordinating Committee. Cooperation has resulted in information exchanges and in numerous visits by scientists of each country to laboratories in the other country.

Japanese fusion research is centered at the JAERI Thermonuclear Fusion Laboratory, Tokai-mura, Japan. The Japanese effort is about one-third the size of the U.S. effort. Major emphasis of the Japanese magnetic confinement program is also on tokamak systems. $(37,38)$ JFT-2 tokamak was completed in March 1972 and was operated in 1973 with an ion temperature of $0.2 \mathrm{keV}$, plasma density of $2 \times 10^{13} \mathrm{~cm}^{-3}$ and energy confinement time of $2 \mathrm{msec}$ with a plasma current of $80 \mathrm{kA}$. Neutral beam heating of the plasma has been studied. A new magnet power supply is being installed to enable an increase in toroidal magnetic field from $10 \mathrm{kG}$ to $18 \mathrm{kG}$.

JFT-2a, a tokamak with teardrop-shaped cross section and an axisymmetric divertor is currently under construction. The Japanese have also begun design work JT-60, a large tokamak device which would confine a plasma at near reactor conditions. They also have a small research 
program related to toroidal pinch experiments, some classified research in laser-fusion, and a strong theoretical and experimental support program centered in a number of Japanese universities.

Formal steps toward international cooperation in fusion research were taken in 1959 by the six countries* of the European Economic Community at the initiative of Euratom. These countries lack the financial and manpower resources necessary to mount self-sufficient rational programs of fusion research similar in scope to those of the United States and the USSR. Under the Euratom plan, a system of "Contracts of Association" between the various national laboratories and Euratom was set up which provided joint funding and joint management of fusion research. (39) Laboratories in Britain and in Denmark have now been added to the Euratom research program. Euratom-sponsored magnetic confinement research has included work on stellarators, mirror machines, theta pinch, z-pinch and tokamak systems.

The management pattern of Euratom-sponsored fusion research is quite complex. Directors of the various laboratories report to steering committees set up within Euratom to oversee the various programs governed by Contracts of Association. In addition, each director must answer to the demands of advisory and program comittees set up by his own national organization for fusion research. In spite of the obvious complexity of the Euratom arrangement, significant research has been accomplished which would not have been possible on a strictly national basis. The Joint European Tokamak (JET), (40) now in the design stage, is an outstanding example of the kind of significant research which is possible as a result of international cooperation.

Within Euratom, an international design team was formed in the autumn of 1973 to plan a large tokamak experiment. The design group, consisting of about 20 individuals, is led by a French scientist from the laboratory at Fontenay-aux-Roses and is headquartered at the British fusion laboratory at Culham. The Joint European Tokamak (JET) would be capable of burning deuterium-tritium at near-reactor conditions. JET is scheduled for operation about 1980.

Success with the tokamak concept developed initially at the Kurchatov Institute in the USSR has led to emphasis being placed on these magnetic confinement devices both in the United States and abroad. On a worldwide basis, four large tokamak machines are currently being planned or are under construction which would provide tests of tokamak scaling laws and would burn deuteriumtritium fuel at near-reactor conditions. These four tokamak experiments are summarized in Table 6 .

Outside the United States, major laser fusion programs have been underway since the mid 1960s at the Lebedev Institute in Moscow and at the Limeil Weapons Research Center in France. The Russian program is comparable in size and scope to the United States program. Smaller orograms have also been initiated more recently in Japan and in West Germany. The status of the Russian program is indicated by a 1974 report by scientists at the Lebedev Institute ${ }^{(41)}$ describing the implosion of a 100 micron radius sphere of deuterated polyethlene to a density of about 30 grams/ $\mathrm{cm}^{3}$. The experiment was said to have resulted in a Lawson number of about $3 \times 10^{14} \mathrm{~cm}^{-3} \mathrm{sec}$ and to have produced a D-T equivalent peak thermonuclear power in excess of 10 megawatts.

ॠBelgium, France, Italy, Luxembourg, Metherlands and West Germany 
Table 6. Large Tokamak Experiments Currently Planned or Under Construction Worldwide

\begin{tabular}{|c|c|c|c|c|c|c|}
\hline $\begin{array}{l}\text { Device } \\
\text { Name } \\
\end{array}$ & Country & $\begin{array}{c}\text { Date of } \\
\text { Initial } \\
\text { Operation } \\
\end{array}$ & $\begin{array}{l}\text { Major } \\
\text { Radius } \\
\end{array}$ & $\begin{array}{l}\text { Plasma } \\
\text { Radius } \\
\end{array}$ & $\begin{array}{l}\text { Plasma } \\
\text { Current }\end{array}$ & $\begin{array}{l}\text { Toroidal } \\
\text { Magnetic } \\
\text { Field } \\
\end{array}$ \\
\hline TFTR & USA & 1981 & $2.7 \mathrm{~m}$ & $90 \mathrm{~cm}$ & $2500 \mathrm{kA}$ & $50 \mathrm{kG}$ \\
\hline JET & Euratom & 1980 & $2.7 \mathrm{~m}$ & $130 \mathrm{~cm}$ & $4800 \mathrm{kA}$ & $60 \mathrm{kG}$ \\
\hline JT-60 & Japan & 1980 & & $100 \mathrm{~cm}$ & $3300 \mathrm{kA}$ & \\
\hline$T-20$ & USSR & 1982 & & $200 \mathrm{~cm}$ & $6000 \mathrm{kA}$ & \\
\hline
\end{tabular}




\section{MANAGEMENT OF FUSION RESEARCH}

\section{PROGRAM OBJECTIVES}

The goal of the United States fusion program is to demonstrate, before the year 2000, safe reliable economically competitive production of fusion power in a Demonstration Reactor that extrapolates readily to commercial reactors.

To achieve the program goal the following milestones must be attained:

- demonstration of scientific feasibility

- demonstration of energy breakeven

- attainment of net energy gain in an operating fusion machine

- construction and operation of a Demonstration Reactor.

For the magnetic confinement concept, the demonstration of scientific feasibility involves the attainment of fusion reactor conditions of temperature, density and plasma confinement time in a device utilizing either a hydrogen or a deuterium-tritium plasma. For laser fusion, the demonstration of scientific feasibility involves the compression and heating of a D-T pellet by laser beams so that a significant number of neutrons are produced as a result of pellet burn.

Demonstration of energy breakeven in a magnetic confinement device involves energy production by fusion in a deuterium-tritium plasma equal to the energy input to confine and heat the plasma. For laser fusion it involves an energy release from pellet burn equal to the laser energy incident on the pellet.

Net energy gain involves the production of usable electrical energy from a fusion reactor employing either the magnetic confinement or laser fusion concept. The energy output from the reactor would exceed the total energy required to maintain and operate the system. A reactor used to demonstrate net energy gain would incorporate many of the components and systems expected to be included in the demonstration reactor.

Attainment of the Demonstration Reactor milestone involves construction and operation of a device which is large enough to fully evaluate fusion power economics and to serve as a basis for commercial fusion power plants.

To achieve the levels of plasma temperature, density and confinement time necessary for net energy production with magnetic confinement devices requires strong magnetic fields and large plasma volumes, both of which are expensive. Consequently, work to date has been with relatively small devices which are not theoretically capable of simultaneous achievement of both the ignition temperature and the $n \tau$ value required for a fusion reactor. Magnetic confinement devices operated since 1970 have, in many instances, achieved the temperatures, densities and confinement times for which they were designed. As shown in Figure 12, several of these devices have approached or exceeded minimum requirements for reactor operation in one or two of these parameters. Much of the empirical information needed to establish laws which predict how systems should be scaled with respect to size, volume and magnetic field in order to achieve reactor conditions has been developed. The simultaneous attainment of all three reactor plasma conditions 
in a single device will require construction of machines which will be much larger than any built to date and which will incorporate many desirable features developed and tested during operation of previous smaller machines.

Achievement of net energy gain in a laser fusion device will require pellet compression to approximately 10,000 times the density of ordinary liquids. This is calculated to require laser energies in the 1 Megajoule range. It is probable that present $\mathrm{Nd}-\mathrm{glass}$ and $\mathrm{CO}_{2}-g a s$ lasers cannot be scaled up to this energy and that a new laser will need to be developed. However, present lasers can probably be developed to the point of demonstrating scientific feasibility and possibly energy breakeven. Technical experience gained with smaller systems will be invaluable in the design and construction of laser fusion reactors employing very high energy laser beams.

Demonstration of the feasibility of controlled fusion reactors is a much more complex technical process than was the demonstration of the feasibility of fission reactors. The demonstration of fission utilized existing technology and involved the manipulation of materials which were readily available. Relatively little development work in the areas of technology or of materials science was required for this demonstration. This is clearly not the case with respect to the fusion reactor demonstration. Because of the extreme physical conditions imposed by fusion requirements, many sophisticated technical and materials problems have had to be solved to progress to the present stage, and other problems must be addressed before fusion reactor feasibility experiments are successful. Examples of fusion related problens on which some work has been done, but much remains to be accomplished, include superconducting magnet development, tritium handing and storage, radiation damage to materials, development of laser amplifiers and optical delivery systems, and reactor coolant technology.

The requirement of parallel solution to both confinement and technological problems imposes different conditions with regard to time and funding on the fusion program than were evident for the fission program. It also means that significant progress toward the solution of many key fusion reactor technology problems will have been made at the time that fusion reactor feasibility is demonstrated.

Planning documents $(42,43,44)$ prepared by the Energy Research and Development Administration provide details of programs leading to achievement of a Demonstration Reactor before the year 2000. Both WASH-1290 ${ }^{(43)}$ and WASH-1363 ${ }^{(44)}$ focus on near term programs (to 1980) which will demonstrate scientific feasibility and provide justification for the long term programs involving construction of large machines for net energy production. A more recent document ${ }^{(42)}$ published by the Division of Magnetic Fusion Energy updates the near term magnetic confinement program and describes alternative paths to achievement of a Demonstration Reactor.

\section{SPONSORSHIP OF FUSION RESEARCH}

The primary source of fusion research support in the United States is the federal government through the Energy Research and Development Administration (ERDA). Reasons for federal support of fusion research include the following: 
- The total cost of fusion power research and development is far beyond that which private industry could be expected to sustain both in terms of the dollar amount and the long development time and financial risk involved.

- Successful development of fusion power would have important national security and foreign trade implications because the country would be self sufficient as far as fusion fuel is concerned.

- Successful development of fusion power would have, in addition to commercial applications, many important military and space applications for the nation.

Within ERDA, magnetic confinement research is managed by the Division of Magnetic Fusion Energy (DMFE) (formerly the Division of Controlled Themonuclear Research) and inertial confinement research is managed by the Division of Laser Fusion (DLF) (formerly a branch of the Division of Military Application).

The growth of federally funded fusion programs is shown in Table 7 and Table 8 which provide budget data for the magnetic confinement and inertial confinement programs. The tables show that federal sponsorship of fusion research was maintained at a fairly constant dollar level during the 1960s, but that it has increased significantly during the past five years. Estimated federal budgets for fusion research for FY 1977 are approximately $\$ 227$ million for magnetic confinement research and approximately $\$ 85$ million for inertial confinement research. (45) The total cost of achievement of a Demonstration Reactor by the late 1990 s has been estimated at $\$ 15$ billion. (42)

Approximately 75 percent of the ERDA budget for magnetic confinement reserach is allocated to four laboratories: tokamak research at Oak Ridge National Laboratory (ORNL) and at Princeton Plasma Physics Laboratory (PPPL), theta pinch research at Los Alamos Scientific Laboratory (LASL), magnetic confinement systems is carried out at General Atomic Company, Massachusetts Institute of Technology, United Technology Research Center, the Naval Research Laboratory, and at several university laboratories.

ERDA-sponsored research in laser fusion is carried out at three laboratories: Lawrence Livermore Laboratory (LLL), Los Alamos Scientific Laboratory (LASL), and Sandia Laboratory (SLA). These laboratories in turn support small programs at the Naval Research Laboratory and at a few universities.

Because of the classified nature of laser fusion research, there had been, until 1975, no direct ERDA support of this research at industrial and university laboratories. In February 1975, the Energy Research and Development Administration signed a contract with KMS Fusion, Inc., whereby KMSF would use its laser and target irradiation facilities to perform a series of lasertarget interaction experiments for ERDA. The information obtained from these experiments is to be used to check computer codes presently used for laser fusion calculations. ERDA has also announced plans to participate financially in the develooment of a Nd-glass laser system at the Laboratory for Laser Energetics of the University of Rochester. 
Table 7. Federal Budgets for Magnetic Conf inement Research for the Years 1951 - 1976. (Data from Reference 45)

\begin{tabular}{|c|c|}
\hline Fiscal Year & $\begin{array}{c}\text { Budget } \\
\text { (millions of dollars) }\end{array}$ \\
\hline $1951-1953$ & 1.1 \\
\hline 1954 & 1.8 \\
\hline 1955 & 6.1 \\
\hline 1956 & 7.4 \\
\hline 1957 & 11.6 \\
\hline 1958 & 29.2 \\
\hline 1959 & 28.9 \\
\hline 1960 & 33.7 \\
\hline 1961 & 30.0 \\
\hline 1962 & 24.8 \\
\hline 1963 & 25.5 \\
\hline 1964 & 22.6 \\
\hline 1965 & 23.1 \\
\hline 1966 & 23.1 \\
\hline 1967 & 23.9 \\
\hline 1968 & 26.6 \\
\hline 1969 & 29.7 \\
\hline 1970 & 34.3 \\
\hline 1971 & 32.2 \\
\hline 1972 & 33.3 \\
\hline 1973 & 39.1 \\
\hline 1974 & 56.4 \\
\hline 1975 & 92.7 (est.) \\
\hline 1976 & 184.0 (est.) \\
\hline
\end{tabular}

Other government sponsored fusion research, amounting to several million dollars per year, has included NASA-sponsored work on heating magnetically contained plasma and on laser development, and plasma physics programs at the Naval Research Laboratory, the National Bureau of Standards, and at colleges and universities where a prime source of funds has been the National Science Foundation. Magnet and cryogenic research has been sponsored by the National Bureau of Standards, the Department of Defense, the National Aeronautics and Space Adminstration, and the National Science Foundation. The Department of Defense is a prime sponsor of research and development of high-energy short-pulse laser systems.

There are a number of significant private fusion research programs. These include both magnetic confinement and laser fusion programs sponsored by the Electric Power Research Institute; contributions to joint programs with the Energy Research and Development Administration from General Atomic, United Aircraft, and Westinghouse; and laser fusion programs at KMS Industries, the University of Rochester and Battelle Memorial Institute. 
Table 8. Federal Budgets for Inertial Confinement Research for the Years 1963 - 1976 (Data from Reference 45)

\begin{tabular}{cc} 
Fiscal Year & $\begin{array}{c}\text { Budget } \\
\text { (millions of dollars) }\end{array}$ \\
\hline 1963 & 0.2 \\
1964 & 1.1 \\
1965 & 1.3 \\
1966 & 1.2 \\
1967 & 1.4 \\
1968 & 1.3 \\
1969 & 2.1 \\
1970 & 3.2 \\
1971 & 9.4 \\
1972 & 17.9 \\
1973 & 34.2 \\
1974 & 42.6 \\
1975 & 53.9 (est.) \\
1976 & 90.0 (est.)
\end{tabular}

\section{PROGRAM PLANS}

A recent planning document ${ }^{(42)}$ for the magnetic confinement program describes al ternative plans for achievement of a Demonstration Fusion Power Reactor (DEMO) by about the year 2000. Five program options (Logics) are discussed in the planning document. The reference option (Logic III) assumes initial operation of the DEMO in 1998. Other options assume initial operation of the DEMO as early as 1992 or as late as 2007 depending on the level of funding and the reactor concept chosen.

The present magnetic fusion program consists of severa 1 small and medium-sized hydrogen experiments (notably the ORMAK and Alcator tokamaks, the 2X-II B magnetic mirror and the Scyllac theta pinch) and the larger PLT tokamak at Princeton which began operation in 1975. Two other large hydrogen burning tokamaks, Doublet III at General Atomic Company (designed to test operation of a large volume tokamak of noncircular cross section) and POX at Princeton (designed to test divertor concepts for impurity control in tokamaks) are under construction and scheduled to operate in mid 1978.

The tokamak approach to fusion has the highest programatic priority and is believed to be closer to achieving a demonstration reactor for commerical application than other magnetic confinement concepts (magnetic mirror and theta pinch) currently under study. Approximately 60 percent of DMFE administered funds are spent for tokamak related research.

In July 1974, a decision was made that the first D-T burning device in the United States should be a tokamak. The Tokamak Fusion Test Reactor (TFTR) ${ }^{(46)}$ at Princeton University is estimated to cost about $\$ 228$ million. Construction activities have begun and completion of 
construction is scheduled for 1981. After about one year of operation with a hydrogen plasma, the reactor is scheduled to be converted to a deuterfum-tritium plasma. It is anticipated that TFTR will achieve a reactor grade plasma and will thus demonstrate the feasibility of the tokamak concept. Proposed characteristics of the Tokamak Fusion Test Reactor include:

- Vacuum chamber 2.7 m major radius; $1.1 \mathrm{~m}$ minor radius

- 5 Tesla (50 kG) toroidal magnetic field

- $20 \mathrm{MW}, 120 \mathrm{keV}, 0.5 \mathrm{sec}$ pulse duration neutral beams

- ion temperature of 5 to $10 \mathrm{keV}$

- ion density of approximately $10^{14} \mathrm{~cm}^{-3}$

- $n \tau=10^{13}$ to $10^{14} \mathrm{~cm}^{-3} \mathrm{sec}$.

A large D-T burning magnetic mirror experiment, $M X$, has al so been proposed for operation in the early 1980s.

Following proof-of-principle (i.e., demonstration of scientific feasibility) with the tokamak or other concept, the magnetic confinement program outlined in Reference 42 describes three confinement devices leading to commercialization of fusion power. These devices are:

- Prototype Experimental Power Reactor or Ignition Test Reactor (PEPR/ITR)

- Experimental Power Reactor (EPR)

- Demonstration Reactor (DEMO).

Proposed characteristics for these devices are 1 isted in Table 9 . It is planned that each confinement concept for which proof-of-principle is established on a timely basis would result in construction of a PEPR/ITR. Only two EPRs would be constructed (probably based on the tokamak and one other concept). The final step would be the OEMO which would be based on the most promising concept and which would be the prototype for comerical fusion reactors.

An important economic issue for fusion power reactors is the lifetime of materials subjected to the punishing environment of bombardment by $14 \mathrm{Mev}$ neutrons from the D-T reaction. Little is known about the actual structural damage that could result from the high energy neutron bombardment. Estimates of fusion reactor wall lifetimes range from a fraction of a year to several years.

To assess the structural damage to reactor components from prolonged bombardment by very high energy neutrons, a series of experimental devices are planned which will provide intense neutron beams for target irradiation purposes. The first of these faiclities, the Rotating Target Neutron Source (RTNS) is currently under construction at Lawrence Livermore Laboratory and scheduled to be operational in early 1978. The RTNS will provide a $1 \mathrm{~cm}^{3}$ irradiation volume at a neutron flux of $2 \times 10^{13} \mathrm{n} / \mathrm{cm}^{2} \mathrm{sec}$.

To provide the large experimental volume and high neutron flux level required for ultimate testing of reactor components, a Fusion Engineering Research Facility (FERF) is proposed for operation in the late 1980s. FERF would be a D-T burning driven plasma device (i.e., it would require the injection of power for continuous operation) which would provide a reactor level neutron fiux. 
Table 9. Proposed Characteristics of Devices in the Long-Range Magnetic

Confinement Program Leading to Commercialization of Fusion Power

Prototype Experimental Power Reactor or Ignition Test Reactor (PEPR/ITR)

- Reactor grade D-T plasma

- Superconducting magnets

- Moderate length D-T burn ( $\sim$ tens of seconds)

- Provision for refueling and impurity control

- Tritium fuel cycle and tritium containment and cleanup system representative of projected power reactor systems

- Designed for operation and maintenance in a radiation environment

- Cooled shield and capability to demonstrate heat removal and breeding in modular tests.

Experimental Power Reactor (EPR)

- Steady state power generation at tens of MWe net power

- Thermal efficiency 220 percent

- Plant availability in the 20 to 50 percent range

- Complete high temperature blanket system

- Demonstrates reactor tritium fuel cycle including tritium breeding, a reactor fueling system and a tritium containment/cleanup system

- Demonstrates reactor plant maintenance systems

- Capability to accomodate materials testing

- Scaleable to DEMO.

Demonstration Fusion Power Reactor (DEMO)

- Power generation at a few hundred Mwe net

- Plant availability 60 to 80 percent with high reliability

- Fuel breeding 21.0

- Impurity problems controlled

- Thirty year plant life -- capable of being maintained

- First wall materials capable of lifetimes which are consistent with economic scaling

- Optimized thermal storage system to produce steady state power

- Design and operating regime which extrapolates readily to commercial sized reactors. 
It would probably be based on either the tokamak or the magnetic mirror concept. The large irradiation volume and flextble access available with a FERF would also provide for extensive testing of blanket modules, neutral beams, vacuum systems and other engineering features of an operating reactor as well as for damage tests of construction materials.

A demonstration of the scientific feasibility of inertial confinement with existing lasers is the objective of experiments now in progress at both government sponsored and privately funded laboratories in the United States. While there is general optimism that proof-of-principle can be demonstrated with the current generation of lasers, it is believed that the attainment of additional inertial confinement milestones will require development of laser systems with energies that are two or three orders of magnitude greater than those of present systems. (45) Energies breakeven is expected to require laser energies in the range of 10 to 100 kilojoules and net energy gain to require laser energies of about 100 kilojoules. The laser energy needed for a laserfusion demonstration reactor is expected to be in the 1 Megajoule range.

In ERDA sponsored research aimed at the development of high power lasers, two approaches are being used. The first approach is to do the research and development needed to scale existing lasers to energy levels sufficient to achieve energy breakeven and possibly net energy gain. The two laser types being developed are the optically pumped neodymium-glass laser and the electron beam stabilized, electrically pumped $\mathrm{CO}_{2}$ laser. A large multibeam neodymium-glass laser, the 10,000 Joule High Energy Laser Facility (HELF) at Lawrence Livermore Laboratory, and a large mul tibeam $\mathrm{CO}_{2}$ laser, the 100,000 Joule High Energy Gas Laser Facility (HEGLF) at Los Alamos Scientific Laboratory are both scheduled to become operational before 1980.

For a laser-fusion power reactor, it is believed that a laser system delivering about 1 Megajoule with a pulse duration of 0.1 to 1.0 nanoseconds will be required. The laser should operate in the 3000 to 8000 Angstrom wavelength region, and should be capable of delivering 10 to 100 pulses per second. All three ERDA laboratories (LASL, LLL and SLA) are doing research aimed at identifying and developing new laser systems. Systems under active research and development include HF chemical lasers, iodine lasers, oxygen lasers, and xenon, krypton and argon eximer lasers.

Because of the relative newness of the inertial con'finement program and the need for laser development to precede a demonstration of the feasibility of laser-fusion, this concept has not been subject to the same kind of extensive program planning that has gone into the magnetic confinement program. However, current projections indicate a demonstration of proof-of-principle before 1980, wi th the program goal of a laser fusion demonstration reactor in the late 1990s.

The newest program with a goal of attainment of a practical fusion reactor is the electronbeam fusion program. It has been realized for several years that relativistic electron beam technology might be applied to the problem of achieving inertial confinement fusion. However, lack of success in beam focusing tended to discourage serious consideration of this approach to fusion until several experimental breakthroughs to electron beam focusing were made at Sandia Laboratories in 1972.

Present program goals of the electron beam fusion program include understanding the physics of beam focusing and energy deposition in solid media, and eventual attainment of scientific 
breakeven as in the laser-fusion program. An electron beam fusion facility is currently under construction at Sandia Laboratories. The accelerator for this facility will be capable of generating 10 nanosecond bursts of 200 kilojoule electrons. D-T pellet experiments utilizing the electron beam from the accelerator are due to begin in 1978.

Orderly attainment of the goal of cormercial fusion power requires a demonstration of the feasibility and technology of fusion through construction and operation of Test Reactors, Experimental Power Reactors and a Demonstration Power Reactor. In the planning and construction of these reactor systems all applicable regulations relating to the operation of nuclear facilities will be followed. The timetable envisioned for construction of these systems provides ample time for preparation of environmental impact statements and for regulatory review by appropriate agencies. 


\section{REFERENCES}

1. A. S. Bishop, "Project Sherwood, Addison-Wesley Publishing Company, Inc., Reading, MA, 1959.

2. L. Spitzer, Jr., "The Stellarator," Scientific American, 199(4):28, October 1958.

3. T. K. Fowler and R. F. Post, "Progress Toward Fusion Power," Scientific American, 215(6):21, December 1966.

4. F. F. Chen, "The Leakage Problem in Fusion Reactors," Scientific American, 217(1):76, July 1967.

5. A. S. Bishop, "Recent World Developments in Controlled Fusion," Nuclear Fusion, 10(T):85, March 1970.

6. W. C. Gough and B. J. Eastlund, "The Prospects of Fusion Power," Scientific American, 224(2):50, February 1971 .

7. B. Coppi and J. Rem, "The Tokamak Approach in Fusion Research," Scientific American, 227(l):65, July 1972 .

8. R. F. Post, "Prospects for Fusion Power," Physics Today, 26(4):30, April 1973.

9. R. F. Post and F. L. Ribe, "Fusion Reactors as Future Energy Sources," Science, 186(4162):397, November 1, 1974 .

10. R. L. Hirsch, "Status and Future Directions of the World Program in Fusion Research and Development," Annual Review of Nuclear Science, 25:79, 1975.

11. Y. T. Baiborodov, M. S. Ioffe, V. M. Petrov, and R. I. Solbolev, Soviet Journal of Atomic Energy, 14:459, 1964.

12. Statement by Melvin B. Gottlieb, Director, Plasma Physics Laboratory, Princeton University before the Joint Committee on Atomic Energy, July 25, 1973.

13. Statement by Herman Postma, Director of the Thermonuclear Division, Oak Ridge National Laboratory before the Joint Committee on Atomic Energy, July 25, 1973.

14. R. L. Ribe, "The Los Alamos Scyllac Program of High-Beta Controlled Fusion Research," LA-UR-73-1055. Prepared for the July 25, 1973, Hearings on Controlled Thermonuclear Research, before the Joint Committee on Atomic Energy.

15. R. F. Post, "Progress Toward Mirror Fusion Reactors," Lawrence Livermore Laboratory, University of California, Livermore, California, prepared for the JCAE Hearings, July $25,1973$.

16. Reference 16 was not used.

17. J. L. Tuck, "Outlook for Controlled Fusion Power," Nature, 233:593, October 29, 1971.

18. W. D. Metz, "Laser Fusion: A New Approach to Thermonuclear Power, " Science, 177:1180, September 29, 1972.

19. K. Boyer, "Laser-Initiated Fusion: Key Experiments Looming," Astronautics and Aeronautics, I1(1):28, January 1973.

20. J. Nuckolis, J. Enmett and L. Wood, "Laser-Induced Thermonuclear Fusion," Physics Today, 26(8):46, August 1973.

21. J. L. Emmett, J. Nuckolls and L. Hood, "Fusion Power by Laser Implosion," Scientific American, 230(6):24, June 1974 . 
22. L. K. Isaacson, Laser-Fusion Program Summary Report, EPRI SR-9, Electric Power Research Institute, Palo Alto, CA, February 1975.

23. J. M. Dawson, "On the Production of Plasma by Giant Pulse Lasers," Physics of Fluids, $\underline{7}(7)$ : 981, July 1964.

24. N. G. Basov and O. H. Krokhin, Soviet Physics JETP, 19:123, 1964.

25. AEC Laser and Electron Beam Programs, FY 1976 - FY 1980, WASH-1363, U.S. Atomic Energy Commission, July 1974.

26. R. F. Post, Fusion Power: The Transition from Fundamental Science to Fusion Reactor Engineering, UCRL-77055, Lawrence Livermore Laboratory, July 1975.

27. P. J. Mallozzi, et al., "A Multi-Kilojoule Short-Pulse Glass Laser and Its Use in Plasma Heating and X-Ray Production," Paper presented at VIII International Quantum Electronics Conference, June 10-13, 1974, San Francisco, CA.

28. Atomic Energy Clearing House, April 12, 1976.

29. G. Yonas, J. W. Poukey, K. R. Prestwich, J. R. Freman, A. J. Toepfler and M. J. Clauser, "Electron Beam Focusing and Application to Pulsed Fusion," Nuclear Fusion, 14:731, 1974.

30. The Application of High Current Relativistic Electron Beams in Controlled Thermonuclear Research, WASH-1286, Division of Controlled Thermonuclear Research, U.S. Atomic Energy Commission, 1974.

31. M. M. Widner and S. L. Thompson, Sandia Laboratories Report SAND-74-351, 1974.

32. A. J. Toepfler, Bulletin Am. Phys. Soc., 19:856, 1974.

33. World Survey of Major Facilities in Controlled Fusion Research, International Atomic Energy Agency, 1973 Edition, Vienna, 1973.

34. M. K. Romanovskii (ed.), Short Description of the Themonuclear Facilities of the 1. V. Kurchatov Institute for Nuclear Energy, ANL-Trans-952, May 1974.

35. Fusion Forefront, ERDA-DCTR Newsletter, 9(1), March 1976.

36. "Agreement Between US and USSR on Scientific and Technical Cooperation in the Field of Peaceful Uses of Atomic Energy," White House Press Release, June 1973.

37. Third Annual Report of JAERI Thermonuclear Fusion Laboratory, JAERI-M-5564, Japan Atomic Energy Research Institute, Toka i-Mura, Japan, February 1974.

38. Fourth Annual Report of JAERI Thermonuclear Fusion Laboratory, JAERI-M-5888, Japan Atomic Energy Research Institute, Tokai-Mura, Japan, October 1974.

39. R. S. Pease and D. R. Wilson, "International Collaboration in Research on Controlled Thermonuclear Fusion," Contemporary Physics, 15(2):179, 1974.

40. C. M. Braams, et a1., "Joint European Tokamak," Atoomenergia Haar Toepass, 15(12):285, December 1973.

41. L. Wood, Magnetic and Inertial CTR: Present Status and Outlook, UCRL-76573, Lawrence Livermore Laboratory, Livermore, CA, January 1975.

42. Fusion Power by Magnetic Confinement Program Plan, ERDA-76/110/0-4, Division of Magnetic Fusion Energy, U.S. Energy Research and Development Administration, July 1976.

43. Fusion Power by Magnetic Confinement, WASH-1290, Division of Controlled Thermonuclear Research, U.S. Atomic Energy Commission, February 1974. 
44. AEC Laser and Electron Beam Programs: Five Year Plan FY 1976 - FY 1980, WASH-1363, Division of Military Application, U.S. Atomic Energy Commission, July 1974.

45. Efforts to Develop Two Nuclear Concepts That Could Greatly Improve This Country's Future Energy Situation, Report to the Congress by the Comptroller General of the United States, United States General Accounting Office, May 1975.

46. Tokamak Fusion Test Reactor Facilities: Environmental Statement, WASH-1544, U.S. Atomic Energy Commission, Draft, January 1975. 


\section{OFFSITE}

A. A. Churm ERDA Chicago Patent Group

9800 S. Cass Avenue

Argonne, IL 60439

J. W. Beal

ERDA Div. of Magnetic

Fusion Energy

Washington, DC 20545

S. 0. Dean

ERDA Div. of Magnetic

Fusion Energy

Washington, OC 20545

E. E. Kintner

ERDA Div. of Magnetic

Fusion Energy

Washington, DC 20545

J. M. Williams

ERDA Div. of Magnetic Fusion Energy

Washington, DC 20545

J. iv. Grace

ERDA Div. of Magnetic

Fusion Energy

Washington, DC 20545

J. Baublitz

ERDA Div. of Magnetic

Fusion Energy

Washington, DC 20545

3 F. E. Coffman

ERDA Div. of Magnetic Fusion Energy

Washington, DC 20545

J. F. Decker

ERDA Div. of Magnetic

Fusion Energy

Washington, DC 20545

3 K. M. Zwilsky

ERDA Div. of Magnetic

Fusion Energy

washington, DC 20545

Or. Philip M. Stone

ERDA Applied Plasma Physics Program

Washington, DC 20545

G. W. Kuswa

ERDA Div. of Laser Fusion

Washington, DC 20545
R. Blaunstein

ERDA Div. of Biomedical

and Environmental Research

Washington, OC 20545

H. M. Busey

ERDA Div. of Military

Application

Washington, DC 20545

M. A. Bel1

ERDA Div. of Safety

Standards and Compliance

Washington, DC 20545

27 ERDA Technical Information Center

M. S. Kaminsky

Argonne National Laboratory

$9700 \mathrm{~S}$. Cass Avenue

Argonne, IL 60439

V. A. Maroni

Argonne National Laboratory

9700 S. Cass Avenue

Argonne, IL 60439

P. M. Persiani

Argonne National Laboratory

9700 S. Cass Avenue

Argonne, IL 60439

M. Petrick

Engineering and Technology

Division

Argonne National Laboratory

9700 S. Cass Avenue

Argonne, IL 60439

W. E. Parkins, Manager

Atomics International

Component Engineering and

Technology Division

North American Rockwell

P.0. 80x 309

Canoga Park, CA 91304

D. Gurinsky

Brookhaven National Laboratory

ERDA Brookhaven Area Office

Upton, NY 11973

H. J. Kouts

Brookhaven National Laboratory ERDA Brookhaven Area Office

Upton, NY 11973

S. Pearlstein

Brookhaven National Laboratory

ERDA Brookhaven Area Office

Upton, NY 11973

\section{OFFSITE}

J. R. Powell

Brookhaven National Laboratory

ERDA Brookhaven Area Office

Upton, NY 11973

A. J. Impink, Jr.

Carnegie Melion University

Pittsburgh, PA 15213

R. A. Gross

Plasma Research Laboratory

236 SW Mudd Bldg.

Columbia University

New York, NY 10027

W. C. Gough

Electric Power Research Inst.

3412 Hillview Ave.

Palo Alto, CA 94304

G. R. Hopkins

Gulf General Atomic

P.O. Box 1111

San Diego, CA 92112

Zeinab Sabri

Iowa State University

261 Sweeney Hall

Nuclear Engineering Department Ames, IA 50010

R. Borg

Lawrence Livermore Laboratory

P. O. Box 808

Livermore, CA 94550

$T$. K. Fowler

Lawrence Livermore Laboratory

P.0. Box 808

Livermore, CA 94550

R. Moir

Lawrence Livermore Laboratory

P. 0. Box 808

Livermore, CA 94550

A. Carl Haussmann

Lawrence Livermore Laboratory P. $0.80 \times 808$

Livermore, CA 94550

J. Hovingh

Lawrence Livermore Laboratory

P.0. Box 808

Livermore, CA 94550

R. F. Post

Lawrence Livermore Laboratory

P.0. Box 808

Livermore, CA 94550

C. J. Taylor

Lawrence Livermore Laboratory P.0. Box 808

Livermore, CA 94550 
R. Werner

Lawrence Livermore

Laboratory

P.0. Box 808

Livermore, CA 94550

L. L. Wood

Lawrence Livermore

Laboratory

P.0. Box 808

Livermore, CA 94550

W. Bauer

Division Supervisor

of Physical Research

Sandia Labs Livermore

Livermore, CA 94550

L. Booth

Los Alamos Scientific Laboratory

CTN Research

P.0. Box 1663

Los Alamos, NM 87544

D. J. Dudziak

Los Alamos Scientific

Laboratory

CTN Research

P.0. Box 1663

Los Alamos, NM 87544

D. B. Henderson

Los Alamos Scientific

Laboratory

CTN Research

P.0. Box 1663

Los Alamos, NM 87544

E. L. Kemp

Los Alamos Scientific

Laboratory

CTN Research

P.0. Box 1663

Los Alamos, NM 87544

F. L. Ribe

Los Alamos Scientific

Laboratory

CTN Research

P.0. Box 1663

Los Alamos, NM 87544

L. Stewart

Los Alamos Scientific

Ladooratory

CTN Research

P.0. Box 1663

Los Alamos, NM 87544

K. Thomassen

Los Alamos Scientific

Laboratory

CTN Research

P. O. Box 1663

Los Alamos, NM 87544
0 . K. Harling

Massachusetts Institute of

Technology

Cambridge, MA 02139

Bruno Coppi

Department of Physics

Massachusetts Institute of

Technology

Cambridge, MA 02139

L. Lidsky

Dept. of Nuclear Engineering

Massachusetts Institute of

Technology

Cambridge, MA 02139

Norm Rasmussen

Dept. of Nuclear Engineering

Massachusetts Institute of

Technology

Cambridge, MA 02139

David Rose

Massachusetts Institute of

Technology

Cambridge, MA 02139

R. E. Stickney

Mechanical Engineering

Massachusetts Institute of

Technology

Cambridge, MA 02139

J. J. Reinmann

NASA - Lewis Research Center

2100 Bookpark Rd.

Cleveland, $\mathrm{OH} \quad 44135$

Vincent Arp

National Bureau of Standards

Cryogenics Division

Boulder, CO 80302

J. F. Clarke

Oak Ridge National

Laboratory

P.O. Box Y

Oak Ridge, TN 37830

A. P. Fraas

Oak Ridge National

Laboratory

P. O. Box $Y$

Oak Ridge, TN 37830

J. Rand MeNally, Jr.

Oak Ridge National

Laboratory

P. 0. Box Y

Oak Ridge, TN 37830

D. Steiner

Oak Ridge National

Laboratory

P.O. Box Y

Oak Ridge, TN 37830
OFFSITE

J. $S \cot t$

Oak Ridge National Laboratory

P. O. Box $X$

Oak Ridge, TN 37830

3. Banford

Physics International

2700 Merced St.

San Leandro, CA 94577

R. A. Huse

Public Service Electric

and Gas Co.

80 Park Place

Newark, NJ 07101

M. Gottlieb

Princeton University, PPPL

P.0. Box 451

Princeton, NJ 08540

R. G. Mills

Princeton University

P.0. Box 451

Princeton, NJ 08540

E. C. Tanner

Princeton University

P.0. Box 451

Princeton, NJ 08540

H. Perkins

Dept. of Chemistry

Princeton University

Princeton, NJ 06540

R. E. Gold

303 Sayre Hall

Forrestal Campus

P.0. Box 451

Princeton, NJ 06540

M. Kristiansen

Texas Tech. University

Lubbock, TX 79409

A. F. Haught

United Aircraft Research Lab.

United Aircraft Corporation

East Hartford, CT 06108

L. Levine

U.S. Navai Research Laboratory

Washington, DC 20390

C. 2. Serpan, Jr.

U.S. Naval Research Laboratory

Washington, OC 20390

Francis Chen

University of California

Electronics Research

Laboratory

College of Engineering

Berkeley, CA 94720 
A. J. Lichtenberg University of California

Electronics Research Laboratory

College of Engineering

Berkeley, CA 94720

Dave Okrent

U.C.L.A.

Los Angeles, CA 90024

C. D. Hendricks

University of Illinois

Nuclear Engineering

Laboratory

Urbana, IL 61801

G. H. Miley

University of Illinois

Nuclear Engineering

Laboratory

Urbana, IL 61801

Terry Kammash

University of Michigan

Nuclear Engineering

Department

Ann Arbor, MI 48105

Dean Abrahamson

University of Minnesota

School of Public

Affairs

Social Science

Building/309

Minneapolis, MN 55455

W. G. Davey

University of Texas

Department of Physics

Austin, TX 78712

E. Linn Draper, Jr. University of Texas

Department of Physics

Austin, TX 78712

W. E. Orummond

University of Texas

Department of Physics

Austin, TX 78712

Abraham Hertzberg

University of Washington

Aerospace Research

Laboratory

316 Guggenheim

Seattle, WA 98105

A. L. Babo

University of Washington

Nuclear Engineering

Department

Seattle, WA 98105

R. Conn University of Wisconsin Nuclear Engineering

Department

Madison, WI 53706

G. L. Kulcinski

University of Wisconsin Nuclear Engineering

Department

Madison, WI 53706

C. W. Maynard University of Wisconsin Nuclear Engineering Department

Madison, WI 53706

D. Lichtman

Department of Physics

University of Wisconsin

Milwaukee, WE 53201

E. E. Donaldson Washington State University Deparment of Physics

Pullman, WA 99163

D. D. Mahlum

Division of Biomedical and Environmental Research Washington, OC 20545

J. V. Vanston Engineering Science Building University of Texas Austin, TX 78712

Leslie S. Ramsey 450 North 5 th Street Indiana, PA 15701 
ERDA Richland Operations Office

W. A. Burns

Atlantic Richfield Hanford Company

J. D. Kaser

Hanford Engineering

Development Labs

D. G. Doran

H. H. Yoshikawa

Battelle-Northwest

D. T. Aase

G. S. Allison

T. W. Ambrose

D. G. Atteridge

D. A. Baker

J. L. Bates

M. A. Bayne

E. R. Bradley

J. L. Brimhall

R. L. Brodzinski

R. J. Brouns

L. R. Bunnell

L. L. Burger

S. H. Bush

N. E. Carter

T. D. Chikalla

R. G. Clark

T. L. Criswell

S. D. Dahlgren

M. T. Dana

D. E. Deonigi

R. L. Dillon

D. A. Dingee

P. J. Dionne

B. H. Duane

J. W. Finnigan

J. C. Fox

J. J. Fuquay

J. E. Garnier

R. D. Gastil

B. F. Gore

J. N. Hartley

A. J. Haverfield

$U$. P. Jenquin

A. B. Johnson, Jr.

R. H. Jones

T. J. Kabele

W. S. Kelly

H. E. Kissinger

D. A. Kottwitz

N. Laegried

B. R. Leonard, Jr

D. L. Lessor

H. B. Liemohn

R. C. Lijkala

M. A. Mckinnon

R. F. Manes

R. P. Marshall
Battelle-Northwest - Continued

10 E. S. Murphy

R. D. Neison

D. F. Newman

R. E. Nightingale

D. E. Olesen

L. T. Pedersen

R. T. Perry

D. R. Pratt

L. A. Rancitelli

J. F. Remark

U. S. Renné

R. E. Rhoads

W. D. Richmond

W. F. Sandusky

L. C. Schmid

N. M. Sherer

E. P. Simonen

R. I. Smith

J. K. Soldat

C. W. Stewart

K. B. Stewart

R. W. Stewart

J. A. Strand

D. L. Styris

A. M. Sutey

V. L. Teofilo

G. L. Tingey

M. T. Thomas

R. C. Thompson

L. H. Toburen

T. J. Trapp

R. Wang

R. E. Westerman

L. D. Williams

$10 \mathrm{~J}$. R. Young

M. G. Zimmerman

Technical Publications (BH)

5 Technical Information 